\title{
On the Solution of Large-Scale Mixed Integer Programming Scheduling Models
}

\author{
Sara Velez, Andres F. Merchan, Christos T. Maravelias \\ Department of Chemical and Biological Engineering, University of Wisconsin-Madison \\ 1415 Engineering Dr., Madison, WI 53706, USA \\ E-mail: maravelias@wisc.edu
}

\begin{abstract}
In this paper, we show how four recently developed modeling and solution methods can be integrated to address mixed integer programs for the scheduling of largescale chemical production systems. The first method uses multiple discrete time grids. The second adds tightening constraints that lower bound the total production and number of batches for each task and material based on the customer demand, while the third generates upper bounding constraints based on inventory and resource availability. The final method is a reformulation that introduces a new integer variable representing the total number of batches of a task. We apply the aforementioned methods to large-scale problems with a variety of processing features, including variable conversion coefficients, changeovers, various storage policies, continuous processing tasks, setups, and utilities, using a discrete-time model. We illustrate how these methods lead to significant improvements in computational performance.
\end{abstract}

\section{Introduction}

The goal of this paper is to illustrate how recently developed solution methods for mixed-integer programming (MIP) scheduling models can be applied to address large-scale problems with a range of complex processing constraints often found in practice. Specifically, we explore four methods: (1) a discrete-time model that employs different time grids for each task/unit, material, and utility to reduce the number of binary variables ${ }^{1,2}$; (2) a demand-based back-propagation algorithm for the calculation of parameters used to generate tightening constraints in minimization problems ${ }^{3}$; (3) a time- and inventory-based forward-propagation algorithm to define parameters and tightening constraints in maximization problems ${ }^{4}$ and (4) a reformulation of the basic discrete-time model via the introduction of a new integer variable representing the total number of times each task runs ${ }^{5}$.

In this paper, we show how to extend these methods to account for industrially relevant features and then how to combine them to address large-scale instances. We focus on problems in network production environments, that is, environments where a task can consume or produce multiple materials, the output of multiple batches of the same task can be mixed (batch mixing), the output of a single batch can be consumed by multiple downstream batches of the same or different task (batch splitting), and there are recycle streams. While methods (2) - (4) are applicable to both discrete-time and continuous-time models ${ }^{6}, 7$, we focus on the former because they are more general, can be readily extended to account for a number of processing constraints, and were 
recently shown to be computationally superior to continuous-time models for problems in network environments ${ }^{8}$.

The paper is structured as follows. In section 2 we present background material including the basic discrete-time formulation and extensions for variable conversion coefficients, changeovers, material storage in processing units, continuous processes, and buffer tanks. In section 3, we summarize our solution methods, including a discrete-time multi-grid formulation (section 3.1), the tightening methods based on demand (section 3.2) and time/inventory availability with modifications to include processes with variable conversion coefficients (section 3.3), and a reformulation with an extension for changeovers (section 3.3). Finally, in section 4 we describe three large examples and present computational results for different solution methods. We use lowercase italics for indices, uppercase bold letters for sets, uppercase italics for variables, and lowercase Greek letters for parameters.

\section{Background}

In this section, we present an overview of modeling and solution advances in the area of chemical production scheduling (subsection 2.1), present the basic discrete-time state-task network (STN) model (section 2.2), and then discuss some extensions (subsections $2.4-2.11$ ).

\subsection{Literature Review}

Scheduling problems can be solved using a variety of approaches, including (1) simple dispatching rules; (2) rigorous scheduling algorithms (e.g., algorithm of Carlier and Pinson ${ }^{9}$ ); (3) heuristic scheduling algorithms (e.g., the shifting bottleneck procedure for job-shops ${ }^{10}$ ); (4) general-purpose metaheuristics (e.g., simulated annealing); (4) constraint programming and the associated constrain propagation algorithms ${ }^{11,12}$; (5) timed automata and reachability analysis algorithms ${ }^{13}$, 14; (6) mathematical programming15, 16; and (7) hybrid methods combining two or more of the above ${ }^{17,18}$. In this paper, we focus on mathematical programming and specifically MIP methods.

In terms of problems, chemical production scheduling problems can be classified in terms of, among other attributes, the production environment ${ }^{16,19}$. There are three major environments: (1) sequential, (2) network, and (3) hybrid. In a sequential environment it is assumed that all tasks produce/consume a single material and batch mixing and splitting are not allowed for both the input and output materials. In a network environment tasks can consume and produce multiple materials, and there are no restrictions in the way input and output materials are handled; i.e., multiple batches of one task can be mixed or material produced by a single batch can be consumed by multiple downstream batches of the same or different tasks. Finally, the term hybrid is used to describe processes that are not sequential nor network; e.g., processes where a task consumes and/or produces multiple materials some of which have mixing/splitting restrictions.

The various MIP scheduling models can be grouped primarily in terms of the major entities modeled ${ }^{16}$. To address problems in sequential environments researchers developed the so-called batch-based models where batches are assigned to units and then sequenced to enforce resource constraints ${ }^{20-25}$. Problems in network environments were addressed using material-based models where materials (and material flows and inventories) are explicitly modeled and tracked over 
time 26,27 . Interestingly, sequential environments can be viewed as a special case of the network environment, where (1) all tasks consume/produce a single material and (2) materials are subject to material handling restrictions (no splitting/mixing and no recycling). Based on this insight Maravelias and co-workers developed a modeling framework that enables the representation of problems in all types of production environments, including facilities that consist of subsystems of different types 28, 29. Furthermore, material-based models have been extended to account for a number of processing characteristics and constraints ${ }^{30-33}$. Thus, since they can be used to address problems in all types of environments and account for various processing constraints, materialbased models are the focus of this paper.

Schedule optimization for network processes began with discrete-time models where the time horizon is divided into uniform time intervals $26,27,34$. These models appeared to be intractable for the solvers of the time, so most effort to improve solution times then focused on developing smaller models, primarily models employing continuous time representations, where the location of time points is an optimization decision ${ }^{35}$, 36. Continuous-time models have fewer time points and fewer binary variables, which led to the belief that they could be solved faster. Continuous-time models relying both on a single grid common across all units $37,3839,40$ as well as different grids for each unit 41-43 were developed to reduce the number of time points even further.

In terms of MIP-based solution methods, researchers in the process systems engineering (PSE) community have proposed: (1) tightening methods including preprocessing algorithms for fixing binary variables ${ }^{21,44}$ and generating valid inequalities ${ }^{45}$, as well as the solution of auxiliary LP and MIP models for the generation of valid inequalities 46, 47; (2) reformulations including variable disaggregation 48, 49 and reformulation-linearization 47 techniques; (3) decomposition methods relying on the structure of the network 50 , the hierarchy of decisions 51,52 , the iterative solution of a simpler MIP model 53, Lagrangean relaxation and decomposition 54, 55, and rolling horizon approaches 56,57; and (4) algorithmic enhancements including preprocessing algorithms to generate strong valid inequalities ${ }^{3,29}$, and the use of heuristics ${ }^{58-60}$. Furthermore, researchers have proposed decomposition methods that rely on the integration of different solution methods, both for sequential 18,61,62 and network 63,64 environments. Finally, there have been some attempts to design algorithms for distributed and parallel computing 65-67. However, despite the efforts to develop small and tight MIP models as well as effective solution methods, the solution of large-scale scheduling problems in network environments remained challenging until recently.

\subsection{Basic Model}

The structure of the process network is defined in terms of the following sets:

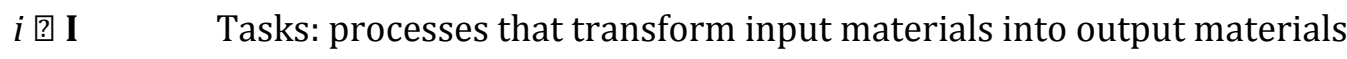

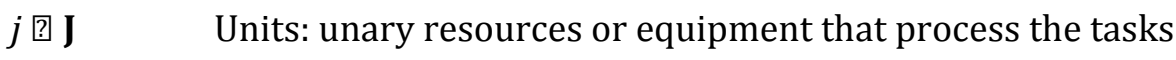

$k$ 目 $\mathbf{K} \quad$ Materials: including feeds, intermediates, and final products

The production environment is defined in terms of the following sets:

$\mathbf{I}_{k}^{+} / \mathbf{I}_{k}^{-} \quad$ tasks producing/consuming material $k$

$\mathbf{I}_{j} \quad$ tasks that can occur in unit $j$

$\mathbf{J}_{i} \quad$ units that can process task $i$

$\mathbf{K}_{i}^{+} / \mathbf{K}_{i}^{-} \quad$ materials produced/consumed by task $i$ 
$\mathbf{K}^{F}$

final products

and the following parameters:

$\beta_{i j}^{\min } / \beta_{i j}^{\max }$ minimum/maximum batch size of task $i$ in unit $j$

$\rho_{i k} \quad$ conversion coefficient of material $k$ produced $(>0)$ or consumed $(<0)$ by task $i$

$\bar{\tau}_{i j} \quad$ (fixed) processing time for task $i$ in unit $j$

$\gamma_{k} \quad$ maximum amount of material $k$ that can be stored

$\xi_{k t} \quad$ amount of material $k$ delivered at time $t(>0)$ or due to customers at time $t(<0) ; \xi_{k 0}$ is the initial inventory of material $k$.

We start with the basic discrete-time MIP formulation of Shah et al.25 Time points are indexed by $t$ and have a uniform spacing of $\delta$ time units, which means time point $t$ occurs at time $t \delta$. The formulation includes one family of binary variables:

$X_{i j t}$ is one if unit $j$ processes task $i$ starting at time $t$

and the following non-negative continuous variables:

$B_{i j t}$ batch-size of task $i$ processed in unit $j$ starting at time $t$

$S_{k t}$ inventory of material $k$ at time $t$

The model consists of unit utilization (eq. 1), unit capacity (eq. 2), material balance (eq. 3) and inventory capacity (eq. 4) constraints.

$\sum_{i \in \mathbf{I}_{j}} \sum_{t \geq t-\tau_{i j}+1}^{t} X_{i j t \prime} \leq 1 \quad \forall j, t$

$\beta_{i j}^{\min } X_{i j t} \leq B_{i j t} \leq \beta_{i j}^{\max } X_{i j t} \quad \forall i, j \in \mathbf{J}_{i}, t$

$S_{k t}=S_{k(t-1)}+\sum_{i \in \mathbf{I}_{k}^{+}} \rho_{i k} \sum_{j \in \mathbf{J}_{i}} B_{i j\left(t-\tau_{i j}\right)}+\sum_{i \in \mathbf{I}_{k}^{-}} \rho_{i k} \sum_{j \in \mathbf{J}_{i}} B_{i j t}+\xi_{k t} \quad \forall k, t$

$S_{k t} \leq \gamma_{k} \quad \forall k, t$

Eq. 1 ensures a unit processes at most one task at a time. Eq. 2 makes sure the batch-size of a task is within the unit's minimum and maximum capacities. Eq. 3 is a material balance and is used to keep track of the production and consumption of each material. Eq. 4 ensures the inventory level is below the storage capacity.

\subsection{Modeling of Time}

Discrete-time models are very general and allow the modeling of various processing features seamlessly. First, unlike their continuous time counterparts, they result in linear modeling of holding (Figure 1A) and utility costs (Figure 1B) because the length of each time period is known. Second, time-varying resource availability and pricing (Figure 2) and intermediate deliveries and due dates (Figure 3) are modeled by simply changing parameters, while continuous-time models require additional binary variables to match these events to time points. Third, features such as setups (Figure 4A) and material production during a task (Figure 4B) can be seamlessly modeled. In the next subsections, we present constraints for the process features that are present in our examples. 

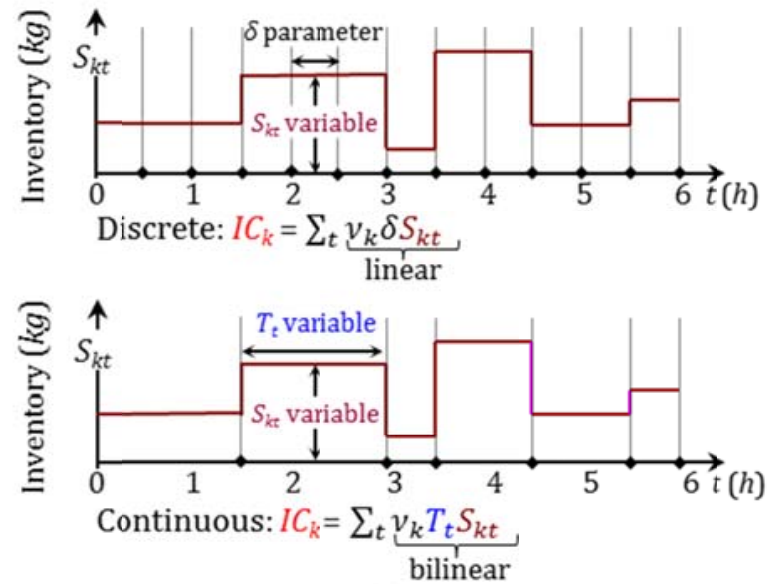

A. Inventory cost, $I C_{\boldsymbol{k}}\left(v_{k}:\right.$ unit cost $\left.[\$ /(\mathrm{kg} \cdot \mathrm{h})]\right)$

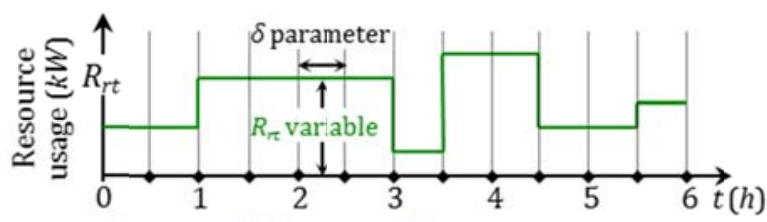

Discrete: $U C_{r}=\sum_{t} \sigma_{r} \delta R_{r t}$

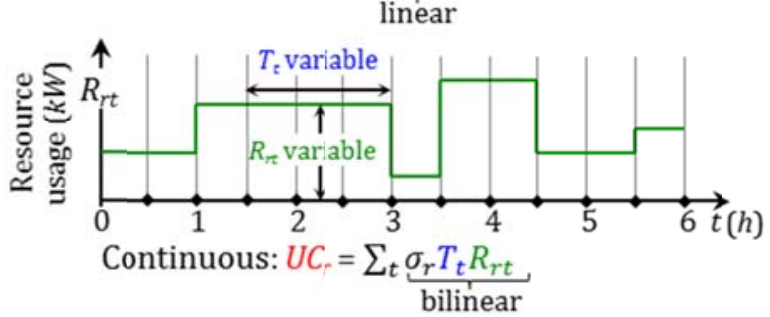

B. Utility cost, $\boldsymbol{U} \boldsymbol{C}_{r}\left(\sigma_{r}:\right.$ unit cost $\left.[\$ /(k W \cdot h)]\right)$

Figure 1. Modeling of inventory and utility costs (modified from Velez and Maravelias ${ }^{68}$ )

$\delta$ = length of discrete period; $S_{k t}=$ inventory of material $k$ levell during period $t$; $T_{t}=$ timing of time point $t$ in continuous-time models; $R_{r t}=$ consumption of utility $r$ during period $t ; v_{k}=$ unit inventory cost for material $k$ $\left(\$ \cdot \mathrm{kg}^{-1} \cdot \mathrm{h}^{-1}\right) ; \sigma_{r}=$ unit utility cost $\left(\$ \cdot \mathrm{kW}^{-1} \cdot \mathrm{h}^{-1}\right) ; I C_{k}=$ total inventory cost for material $k ; U C_{r}=$ total utility cost for utility $r$ (parameters given in black). All types of costs based on time profiles of variables remain linear in discrete-time but become bilinear in continuous-time.

Furthermore, a recent computational study showed that discrete-time formulations are faster, find better solutions within a given time (by 5-16\%), and find solutions with a smaller optimality gap (reducing the gap from about $4-19 \%$ to $2-6 \%$ on average) ${ }^{8}$. A summary of this results is shown in Figure 5. Each line represents the average over 18-24 instances for CPU time or optimality gap. Nevertheless, it is important to note that continuous-time models can be more effective for special classes of problems. For example, when none of the features that lead to nonlinearities or require the introduction of new binaries are present, continuous-time models can be faster. 


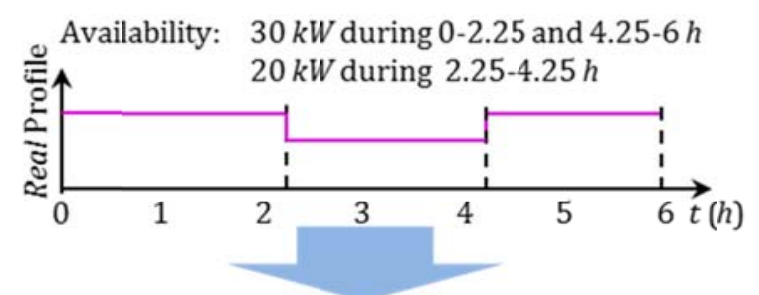

Calculation of time-varying resource availability $\theta_{r t}$

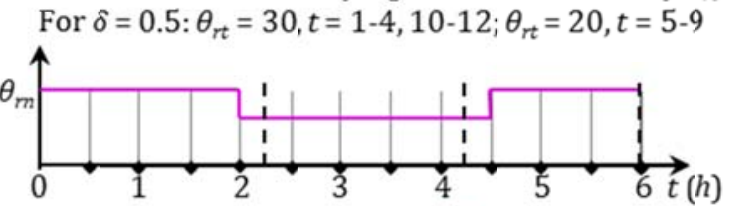

Resource constraint: $R_{r t} \leq \underbrace{\theta_{r t}}_{r t}$

parameter

\section{A. Time-varying resource availability}

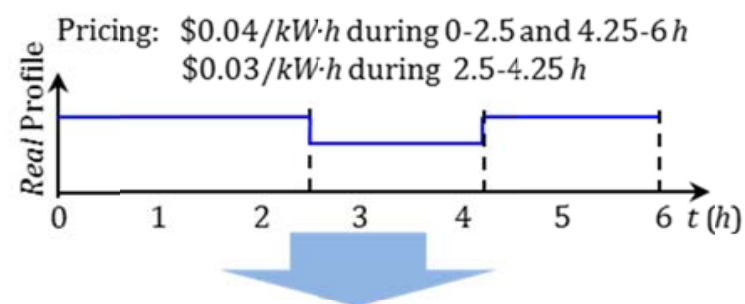

Calculation of time-varying resource price $\sigma_{r t}$

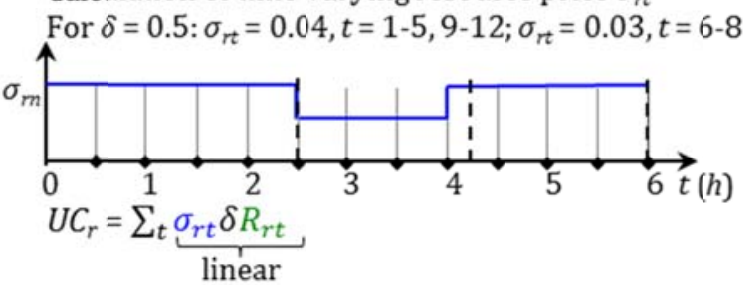

B. Time-varying resource pricing

Figure 2. Modeling of time-varying costs and resource availability (modified from Velez and Maravelias ${ }^{68}$ )

$\delta$ = length of discrete-time period; $\theta_{r t}=$ availability of resource $r$ during period $t ; R_{r t}=$ consumption of utility $r$ during period $t ; \sigma_{r t}=$ price of utility $r$ during period $t$. 
Deliveries:

$20 \mathrm{~kg}$ of A $30 \mathrm{~kg}$ of B

@ $t=0.5 h$ @ t=1.75h

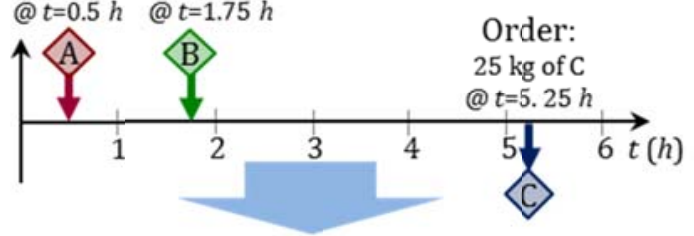

Calculation of shipments $\xi_{k t}(\delta=1)$ :

Deliveries/orders moved to next/previous point if needed

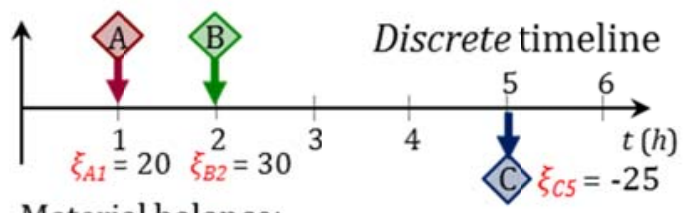

Material balance:

$$
S_{k t}=S_{k, t-1}+\sum_{i, j} \rho_{i k} B_{i j, t-\tau_{i j}}+\sum_{i, j} \rho_{i k} B_{i j t}+\xi_{k t}
$$

Figure 3. Modeling of intermediate deliveries and orders (modified from Velez and Maravelias ${ }^{68}$ ) $\xi_{k t}=$ net shipment of material $k$ at time $t$ ( $>0$ for deliveries, $<0$ for orders).

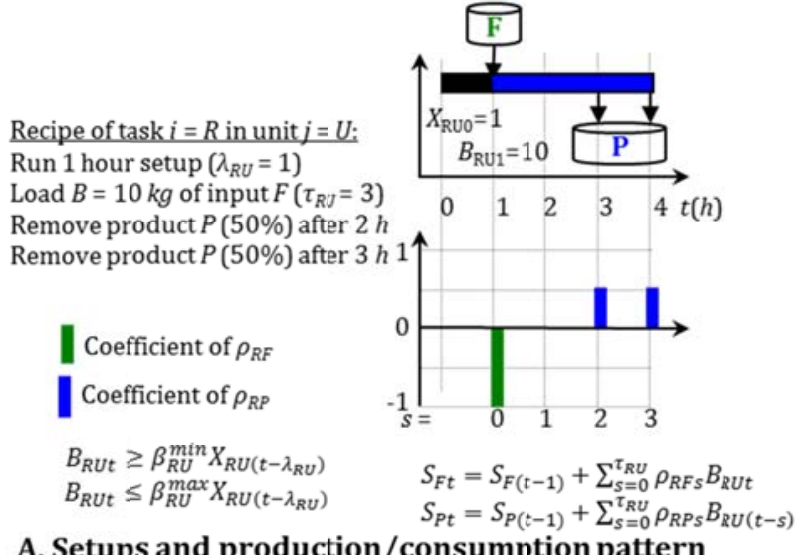

A. Setups and production/consumption pattern

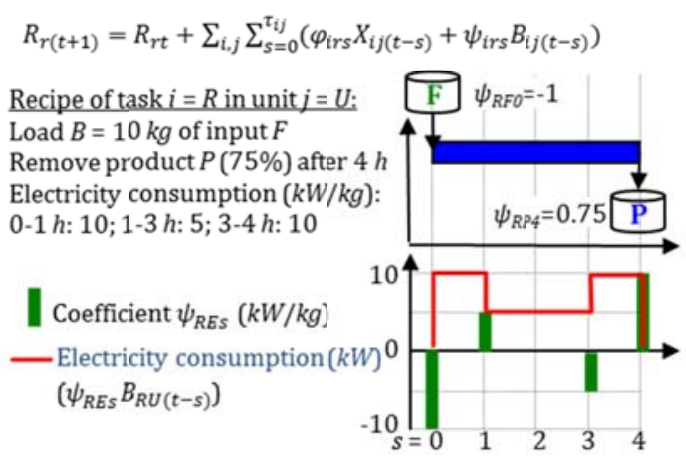

B. Variable resource consumption during task

Figure 4. Modeling of setups, production/consumption pattern, and variable resource consumption during a task (modified from Velez and Maravelias ${ }^{68}$ )

$\lambda_{i j}=$ setup time for task $i$ in unit $j ; \rho_{i k s}=$ conversion coefficient of task $i$ to material $k$ at time point $s$ after the start of task $i . \varphi_{\text {irs }}=$ fixed consumption coefficient of resource $r$ for task $i$ during time period $s$ after the start of task $i(k W) . \psi_{\text {irs }}=$ variable consumption coefficient of resource $r$ for task $i$ during time period $s$ after the start of task $i(\mathrm{~kW} / \mathrm{kg})$. 


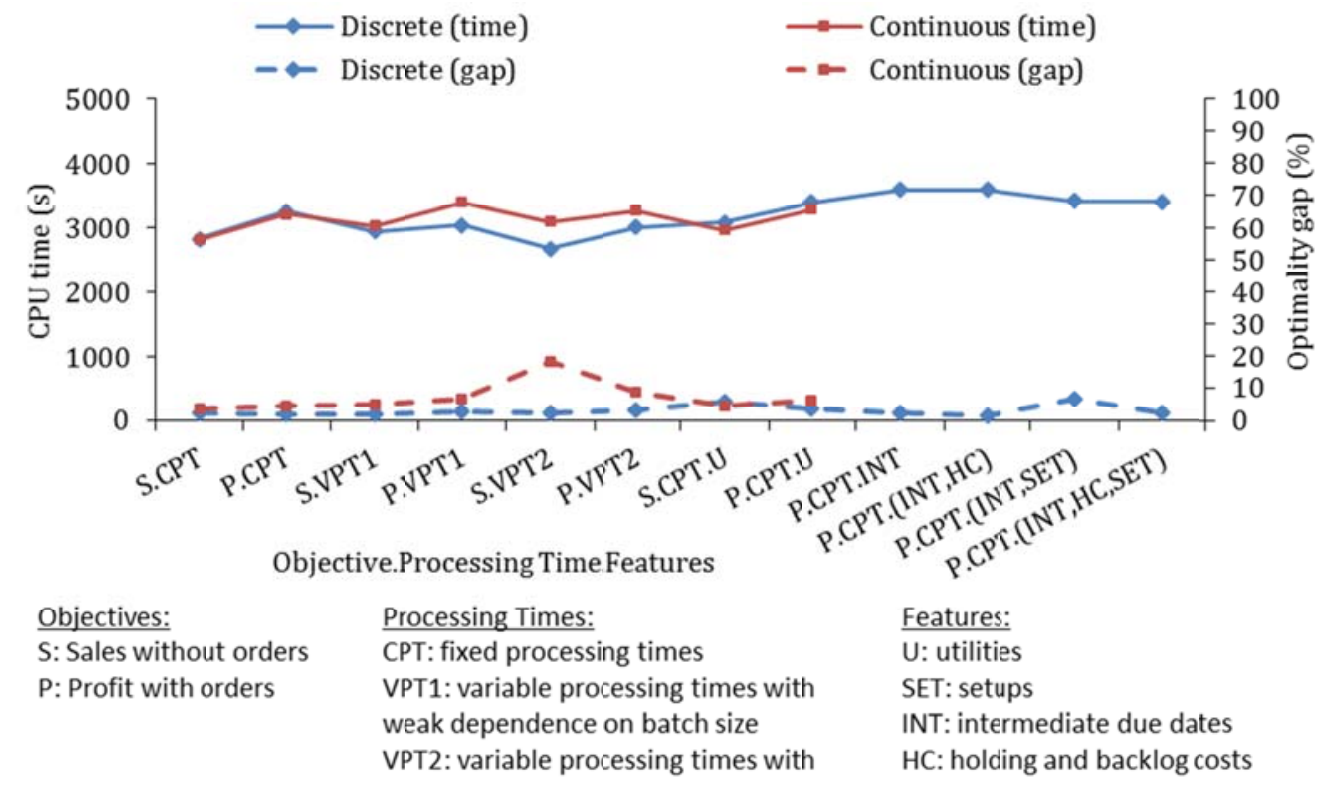

Figure 5. Summary of results of comparative computational study of Sundaramoorthy and Maravelias ${ }^{8}$ Average CPU time and optimality gap for 12 classes of problems labeled as [Objective].[Processing Time].[Features] with discrete and continuous representation of time.

\subsection{Variable Conversion Coefficients}

The material balance (eq. 3) needs to be modified when a task, $i \in \mathbf{I}^{V}$, can produce materials with variable conversion coefficients, where $\rho_{i k}^{\min } / \rho_{i k}^{\max }$ are the minimum/maximum conversion coefficients of task $i$ to material $k$. To model the variable conversions, we introduce a new variable,

$B_{i j k t}^{\mathrm{V}} \quad$ is the amount of material $k$ that is produced by task $i$ in unit $j$ starting at time $t$ and is only defined for $i \in \mathbf{I}^{V}$

The total amount of material produced in a batch must be equal to the batch size of the task (eq. 5) and the fraction of the batch converted to material $k$ must be within the minimum and maximum conversion coefficients (eq. 6). Finally, we modify the material balance so that variable $B_{i j k t}^{\mathrm{V}}$ is included instead of $\rho_{i k} B_{i j t}$ for tasks in $\mathbf{I}^{V}$ (eq. 7).

$$
\begin{aligned}
& \sum_{k \in \mathbf{K}_{i}^{+}} B_{i j k t}^{\mathrm{V}}=B_{i j t} \quad \forall i \in \mathbf{I}^{V} \\
& \rho_{i k}^{\min } B_{i j t} \leq B_{i j k t}^{\mathrm{V}} \leq \rho_{i k}^{\max } B_{i j t} \quad \forall i \in \mathbf{I}^{V}, k \in \mathbf{K}_{i}^{+} \\
& S_{k t}=S_{k(t-1)}+\sum_{i \in \mathbf{I}_{k}^{+} \backslash \mathbf{I}^{V}} \rho_{i k} \sum_{j \in \mathbf{I}_{i}} B_{i j\left(t-\tau_{i j}\right)}+\sum_{i \in \mathbf{I}_{k}^{+} \cap \mathbf{I}^{V}, j \in \mathbf{I}_{i}} B_{i j k\left(t-\tau_{i j}\right)}^{\mathrm{V}}+\sum_{i \in \mathbf{I}_{k}^{-}} \rho_{i k} \sum_{j \in \mathbf{I}_{i}} B_{i j t}+\xi_{k t} \quad \forall k, t
\end{aligned}
$$

\subsection{Changeovers}

Changeovers occur when, for example, cleaning is required between two tasks belonging in two different families. A family $p$ is defined as a subset of tasks that process intermediates or products with similar characteristics (e.g. color, toxicity, corrosivity, etc.) and therefore share similar setup 
requirements. Two new sets are defined to characterize these families. First, the tasks that belong to family $p$ can be grouped in the set $\mathbf{I}_{p}$. Second, the families that can be processed in a particular unit $j$ define the set $\mathbf{P}_{j}$. The parameter $\sigma_{p p^{\prime} j}$ defines the length of the changeover from family $p$ to family $p^{\prime}$ in unit $j$. To allow changeover costs to be modeled, we use a new binary variable,

$Z_{p p^{\prime} j t}$, which is one if there is a changeover from family $p$ to family $p^{\prime}$ in unit $j$ beginning at time $t$.

To enforce changeovers, we use a resource material balance to track which families can be processed by a unit at a time point.

$$
\begin{aligned}
R_{p j t}=\left.R_{p j}^{i n i t}\right|_{t=0} & +\left.R_{p j(t-1)}\right|_{t>0}+\sum_{i \in \mathbf{I}_{p} \cap \mathbf{I}_{j}}\left(X_{i j\left(t-\tau_{i j}\right)}-X_{i j t}\right) \\
& +\sum_{p \in \mathbf{P}_{j} \backslash\{p\}}\left(Z_{p^{\prime} p j\left(t-\sigma_{p^{\prime} p j}\right)}-Z_{p p \prime j t}\right) \quad \forall j \in \mathbf{J}^{C O}, p \in \mathbf{P}_{j}, t
\end{aligned}
$$

where $\mathbf{J}^{C O}$ is the set of units with changeovers, $\mathbf{P}_{j}$ is the set of task families that can be processed by unit $j, R_{p j t}$ is one if unit $j$ is available to start a task belonging to family $p$ at time $t$, and $R_{p j}^{\text {init is a }}$ binary variable that is one if unit $j$ is initially ready for family $p$. The first summation indicates unit $j$ for family $p$ is consumed when a task from family $p$ begins and is released when the task ends. When there is a changeover $\left(Z_{p p \prime j t}=1\right)$, unit $j$ becomes unavailable for family $p$ at time $t$ and becomes available for family $p^{\prime}$ at the end of the changeover. We also need to enforce that unit $j$ can only be available for one family at the beginning of the horizon.

$\sum_{p \in \mathbf{P}_{j}} R_{p j}^{\text {init }}=1 \quad \forall j \in \mathbf{J}^{C O}$

When the changeover from some family $p$ to $p^{\prime \prime}$ is longer than the changeover from $p$ to $p^{\prime}$ plus the changeover from $p^{\prime}$ to $p^{\prime \prime}\left(\exists p, p, p \quad: \sigma_{p p^{\prime \prime}} \geq \sigma_{p p^{\prime}}+\sigma_{p^{\prime} p^{\prime \prime}}\right)$, eq. 8 may schedule a changeover from $p$ to $p^{\prime}$ and from $p^{\prime}$ to $p^{\prime \prime}$ without running any task $i \in \mathbf{I}_{p} \quad$ in order to avoid the longer changeover from $p$ to $p^{\prime \prime}$. To prevent this shortcut, it is necessary to ensure that a task from family $p$ begins after the changeover to that family. We require the task from family $p$ to begin immediately after the changeover to family $p$ ends to remove some of the flexibility for when the changeover occurs, which reduces the number of equivalent schedules. We have found that adding eq. 10 improves the solution times significantly even when it is not strictly required.

$$
\sum_{i \in \mathbf{I}_{p} \cap \mathbf{I}_{j}} X_{i j t} \geq \sum_{p \in \mathbf{P}_{j} \backslash\{p\}} Z_{p^{\prime} p j\left(t-\sigma_{p^{\prime} p j}\right)} \forall j \in \mathbf{J}^{C O}, p \in \mathbf{P}_{j}, t
$$

where $\mathbf{I}_{p}$ is the set of tasks belonging to family $p$. For units that do not have changeovers, we continue to use eq. 1 . We also want to be able to model the case where some tasks in a unit do not have changeovers with any other tasks, but some tasks do. We modify eq. 1 , so that no task can start during the changeover between other tasks

$$
\sum_{i \in \mathbf{I}_{j}} \sum_{\geq t-\tau_{i j}+1}^{t} X_{i j t}+\sum_{p, p \in \mathbf{P}_{j} t} \sum_{\geq t-\sigma_{p p^{\prime} j t}+1}^{t} Z_{p p^{\prime} j t^{\prime}} \leq 1 \quad \forall j, t
$$


The same formulation is valid if we use individual tasks instead of families, but using families reduces the number of binary variables and constraints. When there are only changeover times and no costs, there are many formulations that do not require binary variables for changeovers ${ }^{26}, 34$.

When changeover costs are sequence-independent, we can avoid the double indexed binary, $Z_{p p^{\prime} j t}$, by using two separate single-index variables:

$Z_{p j t}^{1}$ is one if the changeover from family $p$ (to any other family) starts at time $t$

$Z_{p j t}^{2}$ is one if the changeover to family $p$ (from any other family) ends at time $t$

Eq. 11 is the resource balance where $Z_{p j t}^{2}$ makes unit $j$ available for family $p$ at time $t$ and $Z_{p j t}^{1}$ makes unit $j$ unavailable for family $p$ at time $t$. Eq. 12 ensures $Z_{p j t}^{1}$ and $Z_{p^{\prime} j t}^{2}$ are separated by the changeover time from $p$ to $p^{\prime}$ in unit $j$. If $Z_{p^{\prime} j\left(t-\sigma_{p^{\prime} p j}\right)}^{1}$ is one at time $t$ for any family $p^{\prime}$, then $Z_{p j t}^{2}$ must be one for some family other than $p^{\prime}$.

$\begin{aligned} R_{p j t}=\left.R_{p j}^{i n i t}\right|_{t=0} & +\left.R_{p j(t-1)}\right|_{t>0}-\sum_{i \in \mathbf{I}_{p} \cap \mathbf{I}_{j}} X_{i j t}+\sum_{i \in \mathbf{I}_{p} \cap \mathbf{I}_{j}} X_{i j\left(t-\tau_{i j}\right)}+Z_{p j t}^{2}-Z_{p j t}^{1} \quad \forall j \in \mathbf{J}^{C O}, p \\ & \in \mathbf{P}_{j}, t\end{aligned}$

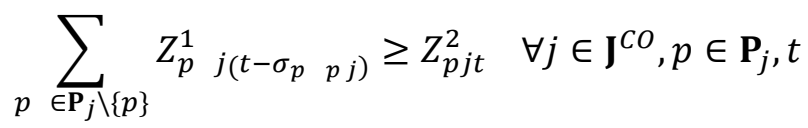

$\sum_{p \in \mathbf{P}_{j} \backslash\{p\}} Z_{p j\left(t+\sigma_{p p} j\right)}^{2} \leq 1 \quad \forall j \in \mathbf{J}^{C O}, p \in \mathbf{P}_{j}, t$

Eq. 12 requires that the changeover and processing times satisfy a triangle inequality: $\sigma_{p p^{\prime} j}+\tau_{i \prime j}+$ $\sigma_{p^{\prime} p^{\prime \prime} j}>\sigma_{p p^{\prime \prime} j} \forall p, p^{\prime}, p^{\prime \prime}, i^{\prime} \in \mathbf{I}_{p^{\prime}}$. Although not necessary for the formulation, we require a task to start as soon as the changeover ends as in eq. 10.

$\sum_{i \in \mathbf{I}_{p} \cap \mathbf{I}_{j}} X_{i j t} \geq Z_{p j t}^{2} \quad \forall j \in \mathbf{J}^{C O}, p \in \mathbf{P}_{j}, t$

\subsection{Continuous Processes}

Continuous processes, $\left(i \in \mathbf{I}^{C}\right)$ are modeled as tasks with processing time of one time period producing their products immediately. We modify the material balance so that material produced by a continuous task is available as soon as the task starts.

$S_{k t}=S_{k(t-1)}+\sum_{i \in \mathbf{I}_{k}^{+} \backslash \mathbf{I}^{C}} \rho_{i k} \sum_{j \in \mathbf{J}_{i}} B_{i j\left(t-\tau_{i j}\right)}+\sum_{i \in \mathbf{I}_{k}^{-} \cup\left(\mathbf{I}_{k}^{+} \cap \mathbf{I}^{C}\right)} \rho_{i k} \sum_{j \in \mathbf{J}_{i}} B_{i j t}+\xi_{k t} \forall k, t$

For continuous processes, $\beta_{j}^{\text {min }} / \beta_{j}^{\max }$ is the minimum $/$ maximum rate $(\mathrm{kg} / \delta \mathrm{hr})$ out of the unit. Continuous processes with residence times can also be modeled by replacing $\tau_{i j}$ in eq. 3 with $\tau_{i j k}^{R}$, where $\tau_{i j k}^{R}$ is the residence time of material $k$ produced by task $i$ in unit $j$. 
We can also enforce that the continuous process must be operating for at least $\tau_{i j}^{\min }$ and no more than $\tau_{i j}^{\max }$ time periods. Eq. 17 enforces the minimum running time by requiring at least $\tau_{i j}^{\min }$ consecutive binaries to be one after the task starts at time $t\left(X_{i j t}=1\right.$ and $\left.X_{i j(t-1)}=0\right)$. Eq. 18 enforces the maximum running time by at least one $X_{i j t}$ to be zero in any time period with $\tau_{i j}^{\max }$ points.

$\sum_{t \leq t} X_{i j t} \geq \tau_{i j}^{\min }\left(X_{i j t}-X_{i j(t-1)}\right) \quad \forall i \in \mathbf{I}^{C}, j \in \mathbf{J}_{i}, t$

$\sum_{t \leq t} X_{i j t} \leq \tau_{i j}^{\max }-1 \quad \forall i \in \mathbf{I}^{C}, j \in \mathbf{J}_{i}, t$

\subsection{Storage in Units}

Material may be temporarily stored inside a processing unit after it is produced. We can model storage in units as well as storage in shared or dedicated storage vessels. Because the examples we consider only have storage in processing units (and no shared or dedicated storage vessels), we can simplify the formulation. To model storage in units, we introduce two new variables:

$S_{k j t}^{P}$ is the amount of material $k$ being stored in processing unit $j$ at time $t$

$F_{k j j \prime}$ is the flow of material $k$ from unit $j$ to $j^{\prime}$ at time $t$

and write a new material balance to track the material stored in each unit. In the material balance, the change in inventory level in processing unit $j$ is equal to the amount produced by a task finishing in the unit minus the amount that flows out of the unit (eq. 19). The amount of material that leaves storage in unit $j$ at time $t$ must be equal to the amount that is consumed by tasks beginning at time $t$ (eq. 20).

$S_{k j t}^{P}=S_{k j(t-1)}^{P}+\sum_{i \in \mathbf{I}_{k}^{+}} \rho_{i k} B_{i j\left(t-\tau_{i j}\right)}-\sum_{j \in \mathbf{J}_{i}} F_{k j j \prime t} \quad \forall k, j \in \mathbf{J}_{k}^{S}, t$

$\sum_{j \in \mathbf{J}_{k}^{S}} F_{k j} j t=\sum_{i \in \mathbf{I}_{k}^{-}}-\rho_{i k} B_{i j t} \quad \forall j, k \in \mathbf{K}_{j}^{-}, t$

where $\mathbf{J}^{S}$ is the set of processing units where storage is allowed, $\mathbf{K}_{j}^{S}$ is the set of materials that can be stored in unit $j, \mathbf{J}_{k}^{S}$ is the set of processing units that can store material $k$, and $\mathbf{K}_{j}^{-}$is the set of materials that can be consumed by unit $j$. Note that we still use the original material balance for all materials. To prevent tasks from starting in unit $j$ while a material is being stored, we set the upper bound on $S_{k j t}^{P}$ to zero whenever a task starts.

$S_{k j t}^{P} \leq \beta_{j}^{\max }\left(1-\sum_{i \in \mathbf{I}_{j}} X_{i j t}\right) \quad \forall j \in \mathbf{J}^{S}, k \in \mathbf{K}_{j}^{S}, t$

These constraints only consider the case where output material can be stored in a processing unit and there are no separate shared storage vessels because that is what is present in the examples we 
consider. It is possible to model the storage of input and output materials in processing units as well as in shared storage vessels using a similar approach ${ }^{29}$.

\subsection{Buffer Tanks}

Buffer tanks $\left(j \in \mathbf{J}^{B}\right)$ are treated as continuous processes with storage in units. Therefore their material balance is

$S_{k j t}^{P}=S_{k j(t-1)}^{P}+\sum_{i \in \mathbf{I}_{k}^{+}} \rho_{i k} B_{i j t}-\sum_{j \in \mathbf{J}_{i}} F_{k j j t} t \quad \forall j \in \mathbf{J}^{B}, k \in \mathbf{K}_{j}^{S}, t$

Also, while material $k$ is being stored in buffer tank $j$, more material $k$ is allowed to enter the buffer tank (but no other materials). We modify eq. 21 so that $S_{k j t}^{P}$ is not forced to zero when a task producing material $k$ begins in a buffer tank.

$S_{k j t}^{P} \leq \beta_{j}^{\operatorname{Smax}}\left(1-\sum_{i \in \mathbf{I}_{j} \backslash \mathbf{I}_{k}^{+}} X_{i j t}\right) \quad \forall j \in \mathbf{J}^{B}, k \in \mathbf{K}_{j}^{S}, t$

where $\beta_{j}^{\text {Smax }}$ is the maximum amount of material that can be stored in unit $j$; for buffer tanks, this may differ from the maximum flow rates in and out.

\subsection{Utilities}

With discrete-time formulations, utilities can be modeled linearly and time-varying utility availability cost and consumption can be modeled by simply changing parameters.

$U_{r t}=\sum_{i, j \in \mathrm{J}_{i}} \sum_{t \geq t-\tau_{i j}+1}^{t} \psi_{i r} B_{i j \mathrm{t}} \leq \theta_{r t} \forall r, t$

where $U_{r t}$ is a continuous variable representing the total amount of resource $r$ used at time $t, \psi_{i r}$ is the amount of resource $r$ used per unit batch size of task $i$ over one time period, and $\theta_{r t}$ is the amount of resource $r$ available at time $t$.

\subsection{Setups}

We consider setups that depend only on the task and the unit, do not have a cost associated with them, and always occur before a task can begin. To model setups, we redefine $X_{i j t}$ slighty: $X_{i j t}$ is one if the setup of unit $j$ for task $i$ begins at time $t$. The definition of $B_{i j t}$ remains the same and is still nonzero only when the task begins after the setup. Based on these definitions, we need to modify eq. 2 to create a lag (equal to the setup time) between when $X_{i j t}=1$ and when $B_{i j t}$ is nonzero.

$\beta_{i j}^{\text {min }} X_{i j\left(t-\lambda_{i j}\right)} \leq B_{i j t} \leq \beta_{i j}^{\max } X_{i j\left(t-\lambda_{i j}\right)} \quad \forall i, j \in \mathbf{J}_{i}, t$

where $\lambda_{i j}$ is the time required to setup unit $j$ for task $i$.

\subsection{Other Extensions}

Material-based discrete-time models have been used to model processes with a wide variety of additional features. Hybrid production environments can be modeled by introducing no-mixing 
and/or no-splitting restrictions on batches of materials ${ }^{29}$. A task can produce or consume multiple materials with different restrictions. Other features include intermediate due and delivery dates; variable utility consumption, price, and availability; pool units ${ }^{30}$; and pipelines ${ }^{30}$. Storage policies such as no intermediate storage, limited storage, and infinite storage can be modeled easily by setting the value of the parameters $\gamma_{k}$ in eq. 4 .

\section{Overview of Solution Methods}

\subsection{Multiple Grids}

Velez and Maravelias ${ }^{1}$ present a method for using multiple discrete-time grids. Each material, task, and unit has its own time grid (Figure 6). The task/unit grid contains points so that

(i) the step size between points is the same for all tasks that can be processed by the same unit,

(ii) the step size is a factor of the processing time so that tasks finish at time points, and

(iii) all grid points for a task producing a material are also in the grid for the tasks consuming the material.

These three requirements ensure that the formulation (1) does not lead to unnecessary idle time between tasks (requirements i and ii) and (2) allows a material to be consumed as soon as it is produced (requirement iii). The three requirements can also be relaxed based on three user defined parameters to reduce the number of time points further. The material grid contains points whenever a material can be produced. Velez and Maravelias ${ }^{1}$ extended the multi-grid formulation to include limited material storage, changeovers, unit maintenance, utilities, and intermediate deliveries. They also proved that the optimal multi-grid solution will be as good as the optimal single-grid solution using an arbitrarily small step size when processing times are independent of batch sizes and resource profiles are piecewise-constant ${ }^{69}$. Their theoretical results also imply that a discrete-time formulation using a single-grid that satisfies the multi-grid conditions is guaranteed to be at least as good as the optimal schedule found by any other formulation, including continuoustime formulations. The multi-gird formulation is not valid when there are continuous processes or when material can be stored in processing units.

\subsection{Tightening Methods Based on Customer Demand}

Velez at el. ${ }^{3}$ proposed a demand propagation algorithm for the calculation of parameters that are used to generate tightening constraints in makespan/lateness and cost minimization problems. In this paper, we generalize the tightening methods from Velez at el. ${ }^{3}$ to account for variable conversion coefficients. The other extensions (changeovers, continuous processing, storage in units, and buffer tanks) do not require any changes to the methods. 

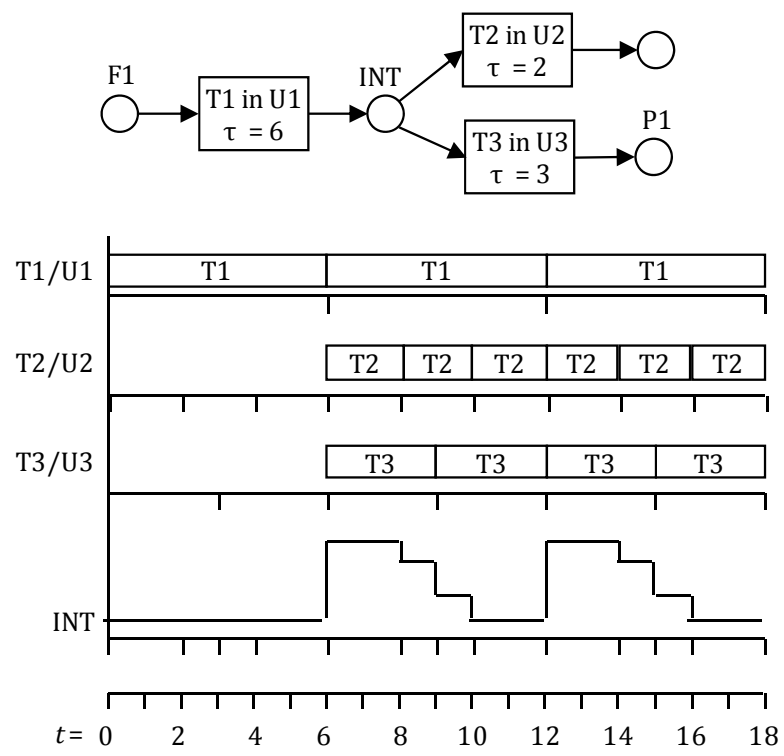

Figure 6. Each task/unit and material has its own grid.

\subsubsection{Overview}

The tightening methods from Velez et al. ${ }^{3}$ add constraints to lower-bound the total number of batches and the total production of each task and each material. A backward propagation algorithm calculates two parameters $\omega_{k}$ and $\mu_{i}$ that are used in the tightening constraints. $\omega_{k}$ is the minimum amount of material $k$ and $\mu_{i}$ is the minimum amount that each task must produce to satisfy customer demand. Figure 7 provides an example of the backward propagation algorithm. First, $\omega_{k}$ is equal to the customer demand for final products: $\omega_{\mathrm{S} 6}=75$ and $\omega_{\mathrm{S} 7}=45$ (step 1; Figure 7c). Next $\mu_{\mathrm{T} 3}=75$ and $\mu_{\mathrm{T} 4}=45$ to produce enough S6 and S7 respectively. The capacity of U4 for T4 is 25$35 \mathrm{~kg}$, which means it can produce $25-35 \mathrm{~kg}$ in one batch, 50-70 kg in two batches, etc. Since it is impossible to produce exactly $45 \mathrm{~kg}$, we can increase the amount produced to $\mu_{\mathrm{T} 4}^{1}=50$ (note that we use $\mu_{i}^{1}$ for the increased amount based on unit capacities). Next we calculate $\omega_{\mathrm{S} 4}=20$ to provide enough material to $\mathrm{T} 4$, and $\omega_{\mathrm{S} 5}=105$ to provide enough material to both $\mathrm{T} 3(75 \mathrm{~kg})$ and $\mathrm{T} 4(30 \mathrm{~kg}) \cdot \mu_{\mathrm{T} 1}=105$ to produce enough $\mathrm{S} 5$, and $\mu_{\mathrm{T} 1}^{1}=105$ based on the unit capacities. $\omega_{\mathrm{S} 3}=21$ to supply enough material to T1. Finally $\mu_{\mathrm{T} 2}=\mu_{\mathrm{T} 2}^{1}=50$ to produce enough of both materials S3 and S5.

When there are no recycle streams and no materials that can be produced by multiple tasks, the backward propagation is straightforward. Velez at al. ${ }^{3}$ present an algorithm that is valid even when there are recycle streams. When materials can be produced my multiple tasks, the value of $\mu_{i}$ must be found by solving a linear program (LP). In section 3.2.2, we present the equations and LP to find $\mu_{i}$ and $\omega_{k}$ based on the modifications to the basic model from section 2. In section 3.3.3, we present the algorithm to calculate $\mu_{i}$ and $\omega_{k}$ (simplified from Velez et al. ${ }^{3}$ ), which is still valid when there are recycle streams and materials produced by multiple tasks. In section 3.2.4, we present the tightening constraints.

\subsubsection{Modifications}

Parameter $\omega_{k}$ is calculated based on the minimum conversion coefficient, $\rho_{i k}^{\min }$, because $\omega_{k}$ will be lowest when the consumption of material $k$ by task $i$ is at its lowest. 
$\omega_{k}=-\sum_{t} \xi_{k t}-\sum_{i \in \mathbf{I}_{k}^{-}} \rho_{i k}^{\min } \mu_{i}^{1}$

Parameter $\mu_{i}$ must be large enough to meet the demand of all materials produced by task $i$. The first term in eq. 26 is the minimum batch size of task $i$ needed for each material and is calculated based on the maximum conversion coefficient, $\rho_{i k}^{\max }$. The second term ensures that $\mu_{i}$ is large enough to meet the total demand of all materials.

$\mu_{i}=\max \left\{\max _{k \in \boldsymbol{K}_{i}^{+}}\left\{\omega_{k} / \rho_{i k}^{\max }\right\}, \sum_{k \in \boldsymbol{K}_{i}^{+}} \omega_{k}\right\}$

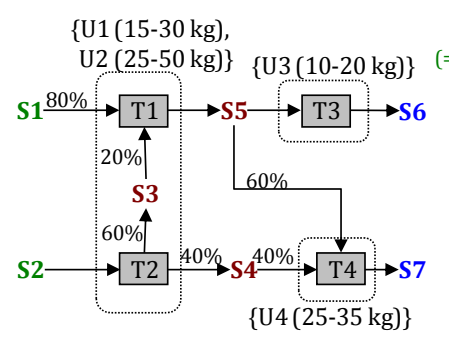

A. Example Network

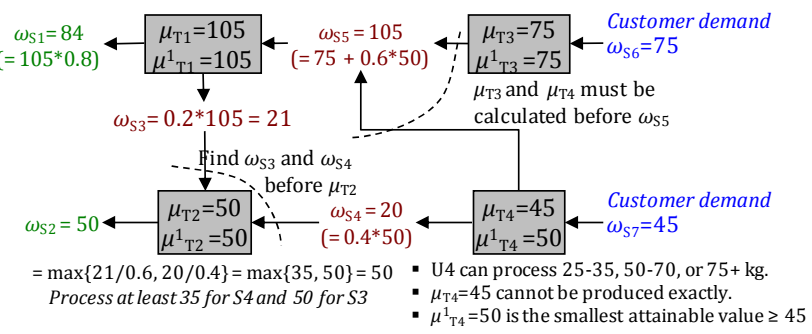

B. Backward propagation algorithm for given demand

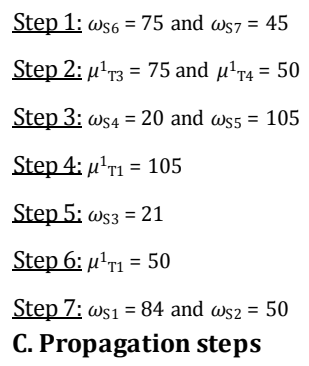

Figure 7. (a) Example network with unit capacities. (b) Calculations in the backward propagation. (c) Steps in the backward propagation

When there are materials that can be produced by multiple tasks, we find $\mu_{i}$ by solving an LP instead of through eq. 27. This LP needs to be modified to allow for variable production amounts following the same method in section 3.2.1.

$\mu_{i}=\min Q_{i}$

s.t. $\quad \sum_{t} \xi_{k t}+\sum_{i \in \mathbf{I}_{k}^{+} \backslash \mathbf{I}^{V}} \rho_{i k} Q_{i}+\sum_{i \in \mathbf{I}_{k}^{+} \cap \mathbf{I}^{V}} Q_{i k}^{V} \geq-\sum_{i \in \mathbf{I}_{k}^{-}} \rho_{i k} Q_{i} \quad \forall k$

$$
\begin{aligned}
& \sum_{i \in \mathbf{I}_{k}^{+} \backslash \mathbf{I}^{V}} \rho_{i k} Q_{i}+\sum_{i \in \mathbf{I}_{k}^{+} \cap \mathbf{I}^{V}} Q_{i k}^{V} \geq \omega_{k} \quad \forall k \\
& \sum_{k \in \mathbf{K}_{i}^{+}} Q_{i k}^{V}=Q_{i} \quad \forall i \quad \in \mathbf{I}^{V} \\
& \rho_{i k}^{\min } Q_{i} \leq Q_{i k}^{V} \leq \rho_{i k}^{\max } Q_{i} \quad \forall i \quad \in \mathbf{I}^{V}, k \in \mathbf{K}_{i}^{+}
\end{aligned}
$$

where $Q_{i}$ is the total amount that task $i$ processes, and $Q_{i k}^{V}$ is the total amount of material $k$ that task $i$ produces. To find $\mu_{i}$, we minimize $Q_{i}$. The first constraint is a material balance that says the amount of material produced/delivered must be greater than the amount that is consumed. The second constraint requires that the amount produced is at least equal to $\omega_{k}$. The third and fourth constraints are analogous to eq. 5 and 6, respectively.

After calculating $\mu_{i}$, we need to determine if it is possible to produce exactly $\mu_{i}$ based on the possible batch sizes for units processing task $i$. We do this by checking all possible combinations of numbers of batches, indexed by $m$, in each unit, where $\varepsilon_{j}^{m}$ is the numbers of batches in unit $j$ in combination $m$. The minimum amount that can be produced by combination $m$ is $\sum_{j \in \mathbf{J}_{i}} \varepsilon_{j}^{m} \beta_{j}^{m i n}$ and the maximum amount is $\sum_{j \in \mathbf{J}_{i}} \varepsilon_{j}^{m} \beta_{j}^{m a x}$. If $\mu_{i}$ is in this range for any value of $m$, then $\mu_{i}^{1}=\mu_{i}$ (line 1 of eq. 28). Otherwise, $\mu_{i}^{1}$ is the smallest amount that can be produced by any combination $m$ whose minimum production is at least $\mu_{i}$ (line 2 of eq. 29). 
$\mu_{i}^{1}=\left\{\begin{array}{cc}\mu_{i} & \exists m: \sum_{j \in \mathbf{J}_{i}} \varepsilon_{j}^{m} \beta_{i j}^{\text {min }} \leq \mu_{i} \leq \sum_{j \in \mathbf{J}_{i}} \varepsilon_{j}^{m} \beta_{i j}^{\text {max }} \\ \min _{m}\left\{\sum_{j \in \mathbf{J}_{i}} \varepsilon_{j}^{m} \beta_{i j}^{m i n}: \sum_{j \in \mathbf{J}_{i}} \varepsilon_{j}^{m} \beta_{i j}^{\text {min }} \geq \mu_{i}\right\} & \text { otherwise }\end{array}\right.$

Note that we can limit the number of combinations we need to check based on the maximum batch size. For each unit we only need to check combinations with up to $\left[\mu_{i} / \beta_{j}^{\max }\right\rceil$ batches. Therefore we consider $m \in\left\{1,2, \ldots, \prod_{j \in \mathbf{J}_{i}}\left(\left[\mu_{i} / \beta_{j}^{\max }\right\rceil+1\right)\right\}$.

\subsubsection{Algorithm}

In this section we present a simplified algorithm for the tightening methods in Velez et al. ${ }^{3}$. Unlike the previous algorithm, this algorithm does not require recycle and tear streams to be identified. However, it may be more expensive and requires solving an LP for all tasks any time a material can be produced by multiple materials. The final output of the two algorithms will be the same.

\section{Tightening Algorithm}

$1 \omega_{k}=0 \forall k ; \mu_{i}=0 \forall i ; \mathbf{K}^{O}=\mathbf{K} ; \mathbf{I}=\mathbf{I}^{O}$

2 While $\left|\mathbf{K}^{O}\right|>0$ or $\left|\mathbf{I}^{O}\right|>0$

3 for $k \in \operatorname{argmin}\left\{\left|\mathbf{I}_{k}^{-} \cap \mathbf{I}^{O}\right|: k \in \mathbf{K}^{O}\right\}$

$4 \quad \mathbf{K}^{O}=\mathbf{K}^{O} \backslash\{k\}$; set $\omega_{k}$ based on eq. 26

5 for $i \in \operatorname{argmin}\left\{\left|\mathbf{K}_{i}^{+} \cap \mathbf{K}^{O}\right|: i \in \mathbf{I}^{O}\right\}$

$6 \quad \mathbf{I}^{O}=\mathbf{I}^{O} \backslash\{i\}$

$7 \quad$ If $\left|\mathbf{K}^{M T}\right|=0$

8 Then set $\mu_{i}$ based on eq. 27

$9 \quad$ Otherwise solve $\mathrm{LP}_{i}$ to find $\mu_{i}$

$10 \quad$ Calculate $\mu_{i}^{1}$ from eq. 28

11 If $\exists k: \sum_{i \in \mathbf{I}_{k}^{+}} \rho_{i k}^{\max } \mu_{i}^{1}<\omega_{k}$

$12 \quad$ Then $\mathbf{K}^{O}=\mathbf{K} ; \mathbf{I}^{O}=\mathbf{I}$

\subsubsection{Constraints}

The constraints bound the number of batches of task (eq. 29) and a material (eq. 30) and the total amount produced by a task (eq. 31) and of a material (eq. 32).

$\sum_{j \in \mathbf{J}_{i}, t} X_{i j t} \geq\left[\mu_{i}^{1} / \max _{j \in \mathbf{J}_{i}}\left\{\beta_{i j}^{\max }\right\}\right] \quad \forall i$

$\sum_{i \in \mathbf{I}_{k}^{+} j \in \mathbf{J}_{i}, t} X_{i j t} \geq\left\lceil\omega_{k} / \max _{i \in \mathbf{I}_{k}^{+} j \in \mathbf{J}_{i}}\left\{\rho_{i k}^{\max } \beta_{i j}^{\max }\right\} \mid \quad \forall k \in \mathbf{K}^{M T}\right.$

$\sum_{j \in \mathrm{J}_{i}, t} \beta_{i j}^{\max } X_{i j t} \geq \mu_{i}^{2} \quad \forall i$

$\sum_{i \in \mathbf{I}_{k}^{+} j \in \mathbf{J}_{i}, t} \rho_{i k}^{\max } \beta_{i j}^{\max } X_{i j t} \geq \omega_{k} \quad \forall k \in \mathbf{K}^{M T}$

where $\mu_{i}^{2}$ is the smallest value greater than $\mu_{i}^{1}$ that is a linear, integer combination of the maximum unit capacities because the LHS of eq. 31, must be a integer combination of the maximum unit capacities. To find $\mu_{i}^{2}$, the same $\varepsilon_{j}^{m}$ from eq. 28 are used.

$\mu_{i}^{2}=\min _{m}\left\{\sum_{j \in \mathbf{J}_{i}} \varepsilon_{j}^{m} \beta_{i j}^{\max }: \sum_{j \in \mathbf{J}_{i}} \varepsilon_{j}^{m} \beta_{i j}^{\max } \geq \mu_{i}\right\}$ 


\subsection{Tightening Methods Based on Resource Availability and Inventory}

Merchan and Maravelias ${ }^{4}$ developed new families of tightening constraints that employ parameters and variables based on (1) the available effective time for each unit and task in the network, and (2) restrictions derived from limited initial inventories of raw materials and intermediates.

\subsubsection{Overview}

The main goal of these new tightening methods is to provide upper bounds to the total number of batches of each task and the total production of each material. These methods are largely based on the concept of effective time window for tasks, $\bar{\theta}_{i j}$ (units, $\bar{\theta}_{j}$ ), defined as the maximum time a task (unit) is allowed to run when considering both the minimum time required to produce its input materials and the minimum time from the end of the horizon to guarantee its output is converted to final products. In principle, these two separate times define two new parameters; the former relates to the Earliest Starting Time, $\bar{\varepsilon}_{i j}\left(\bar{\varepsilon}_{j}\right)$, whereas the latter defines the Shortest Tail from the end of the horizon, $\bar{\sigma}_{i j}\left(\bar{\sigma}_{j}\right)$. Although these parameters have been considered in the past in different contexts, the novelty of this approach is that they are calculated considering not only processing times but also minimum times required to produce every intermediate in the network. Equation (34) shows the relation between these parameters.

$\bar{\theta}_{i j}=\eta-\bar{\varepsilon}_{i j}-\bar{\sigma}_{i j}$

Figure 8 illustrates the calculation of $\bar{\varepsilon}_{i j}$. A similar procedure is followed to calculate $\bar{\sigma}_{i j}$. At time $t=0$, task T1 can start in units $\mathrm{U} 1$ and $\mathrm{U} 2\left(\bar{\varepsilon}_{U 1, T 1}=0\right.$ and $\left.\bar{\varepsilon}_{T 1, U 2}=0\right)$ and task T2 may start in U4 $\left(\bar{\varepsilon}_{T 2, U 4}=0\right)$. At time $t=2, \mathrm{~T} 1 / \mathrm{U} 1$ can produce up to $10 \mathrm{~kg}$ of material S3, while T1/U2 may produce up to $5 \mathrm{~kg}$ of S3 at time $t=3$. At time $t=4, \mathrm{~T} 2 / \mathrm{U} 4$ can produce a maximum of $25 \mathrm{~kg}$ of S 4 and T1/U1 can produce an additional $10 \mathrm{~kg}$ of S3. Since the minimum amount of S3 (25 kg) required to start T3/U3 has been produced, it is established that $\bar{\varepsilon}_{U 3, T 3}=4$. Note that if only processing time were considered, a less tight value of $\bar{\varepsilon}_{U 3, T 3}=2$ would be calculated.

Once the time window has been determined for each task, equation (35) is used to calculate the corresponding time window for each unit.

$\bar{\theta}_{j}=\eta-\min _{i \in \mathbf{I}_{j}} \bar{\varepsilon}_{i j}-\min _{i \in \mathbf{I}_{j}} \bar{\sigma}_{i j}$

Next, a forward propagation algorithm calculates parameters $\bar{\omega}_{k}$ and $\bar{\mu}_{i}$ to respectively define the maximum amount of material $k$ that can be produced and the maximum amount that task $i$ can process within the given horizon when time window and initial inventory restrictions are considered. Figure 9 exemplifies the calculations that occur within this algorithm using the same network introduced in Figure 8. It starts by calculating $\bar{\omega}_{k}$ for materials (raw and intermediates) with initial inventory: $\bar{\omega}_{\mathrm{S} 1}=35$ and $\bar{\omega}_{\mathrm{S} 2}=140$. With these values, $\bar{\mu}_{\mathrm{T} 1}=35$ (T1 is limited by inventory) and $\bar{\mu}_{\mathrm{T} 2}=50$ (T2 is limited by its time window). Propagation to the outputs of T1 and $\mathrm{T} 2$ results in $\bar{\omega}_{\mathrm{S} 2}=35$ and $\bar{\omega}_{\mathrm{S} 4}=50$. Once more, a task is limited by the maximum available inventory, and a value of $\bar{\mu}_{\mathrm{T} 3}=35$ is obtained. However, the capacity of U3 for T3 is $25-30 \mathrm{~kg}$, which means it can process $25-30 \mathrm{~kg}$ in one batch, $50-60 \mathrm{~kg}$ in two batches, and so on, making impossible to process the available $35 \mathrm{~kg}$ of S3. Therefore, the maximum amount that can be processed by T3 is decreased to $\bar{\mu}_{\mathrm{T} 3}^{1}=30$ and a value of $\bar{\omega}_{\mathrm{S} 5}=30$ is obtained. The next step in the 
propagation uses the maximum available inventories of S4 and S5 and the time window for unit U4 to calculate $\bar{\mu}_{\mathrm{T} 4}=50$ and finally, $\bar{\omega}_{\mathrm{S} 6}=50$.

As illustrated in Figure 9, the forward-propagation algorithm is based on the comparison between values predicted for the maximum amount that a task can process from time and inventory availability. The former can be calculated once the time window for the task is known, whereas the latter uses a linear program analogous to the one presented in section 3.2.2 for the demand-based back-propagation algorithm. Equations 36-38 use this comparison to calculate $\bar{\mu}_{i}$. Then, equation 39 is employed to calculate $\bar{\omega}_{k}$. Note that equations 36-39 are valid for the general case of variable conversion coefficients as introduced in section 2.3 .
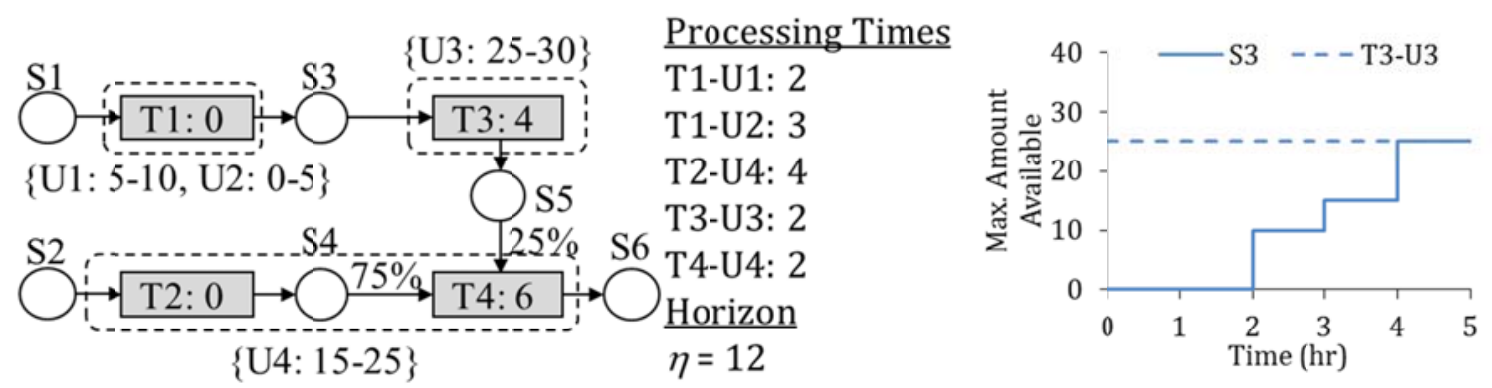

\section{A. Example network}

\section{B. Earliest Start Time calculation}

Figure 8. (a) Example network with processing times and unit capacities. (b) Production history for material $\mathrm{S} 3$ and minimum required S3 to start task T3 in unit U3.

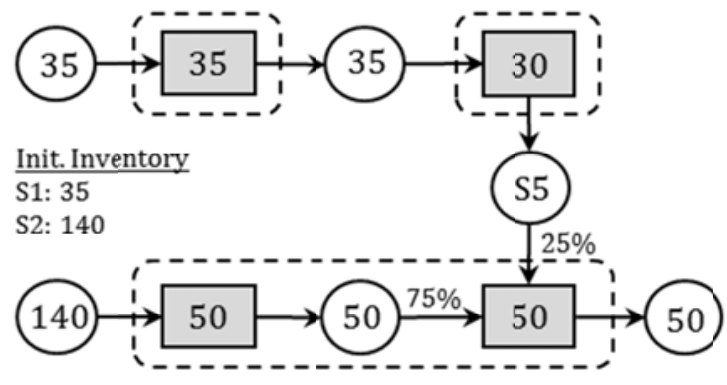

(a) Propagation results

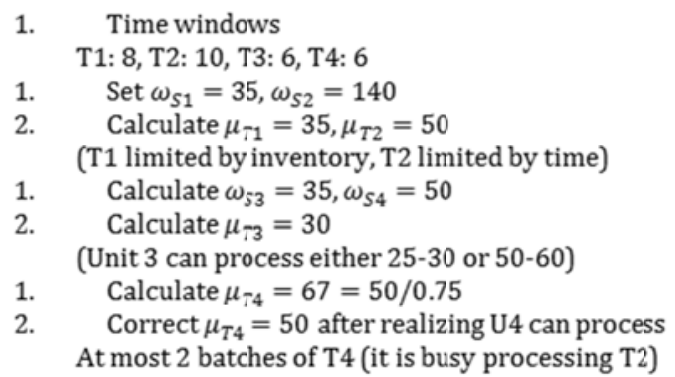

\section{(b) Propagation steps}

Figure 9. (a) Final results after propagation (b) Intermediate steps in the propagation algorithm

$$
\begin{aligned}
& \bar{\mu}_{i}^{T W}=\max _{j \in \mathbf{J}_{i}} \beta_{j}^{\max }\left\lfloor\frac{\bar{\theta}_{i j}}{\bar{\tau}_{i j}}\right\rfloor \\
& \bar{\mu}_{i}^{L P}=\max \left\{\begin{array}{l}
Q_{i}: \xi_{k 0}+\sum_{i^{\prime} \in \mathbf{I}_{k}^{+} \backslash \mathbf{I}^{V}} \rho_{i^{\prime \prime}} Q_{i^{\prime}}+\sum_{i^{\prime} \in \mathbf{I}_{k}^{+} \cap \mathbf{I}^{V}} Q_{i^{\prime} k}^{V}+\sum_{i^{\prime} \in \mathbf{I}_{k}^{-}} \rho_{i^{\prime \prime} k} Q_{i^{\prime}} \geq 0 \quad \forall k \\
\sum_{i^{\prime} \in \mathbf{I}_{k}^{+} \backslash \mathbf{I}^{V}} \rho_{i^{\prime \prime} k} Q_{i^{\prime}}+\sum_{i^{\prime} \in \mathbf{I}_{k}^{+} \cap \mathbf{I}^{V}} Q_{i^{\prime} k}^{V} \leq \bar{\omega}_{k} \forall k \\
\sum_{k \in \mathbf{K}_{i^{\prime}}^{+}} Q_{i^{\prime} k}^{V}=Q_{i^{\prime}}, \forall i^{\prime} \in \mathbf{I}^{V} \\
\rho_{i^{\prime} k}^{\min } Q_{i^{\prime}} \leq Q_{i^{\prime} k}^{V} \leq \rho_{i^{\prime} k}^{\max } Q_{i^{\prime}} \forall i^{\prime} \in \mathbf{I}^{V}, k \in \mathbf{K}_{i^{\prime}}^{+}
\end{array}\right\} \\
& \bar{\mu}_{i}=\min \left\{\bar{\mu}_{i}^{T W}, \bar{\mu}_{i}^{L P}\right\} \\
& \bar{\omega}_{k}=\xi_{k 0}+\sum_{i \in \mathbf{I}_{k}^{+}} \rho_{i k}^{\max } \bar{\mu}_{i}^{1}
\end{aligned}
$$


An analogous procedure to that introduced in section 3.2.2 is followed to determine $\bar{\mu}_{i}^{1}$ by considering the attainable region of each unit, as expressed through equation 40 .

$\bar{\mu}_{i}^{1}=\left\{\begin{array}{cc}\bar{\mu}_{i} & \exists m: \sum_{j \in \mathbf{J}_{i}} \varepsilon_{j}^{m} \beta_{j}^{\text {min }} \leq \bar{\mu}_{i} \leq \sum_{j \in \mathbf{J}_{i}} \varepsilon_{j}^{m} \beta_{j}^{\text {max }} \\ \max _{m}\left\{\sum_{j \in \mathbf{J}_{i}} \varepsilon_{j}^{m} \beta_{j}^{\text {max }}: \sum_{j \in \mathbf{J}_{i}} \varepsilon_{j}^{m} \beta_{j}^{\text {max }} \leq \bar{\mu}_{i}\right\} & \text { otherwise }\end{array}\right.$

After the time windows have been calculated and the forward-propagation has been executed, a new algorithm is used to define the feasible region in which the number of batches of a determined subset of dependent tasks can vary. This groups of dependent tasks are either trivially defined (e.g. tasks sharing the same unit) or identified through a new algorithm based on common upstream tasks and/or materials that render a subset of tasks dependent. Figure 10 illustrates this concept.

For each of the identified groups, which are indexed by $d$, the maximum number of occurrences of each task in the subgroup is calculated using equation (41) that considers restrictions in both the available time and inventory. Subsequently, the set of points for the feasible combinations of the number of batches of each task in the subgroup is determined and the convex hull of these points is obtained by using qhull70. These constraints can directly be introduced to further tighten the formulation as explained in section 3.3.3.

$N_{i d}^{\max }=\min \left\{\sum_{j \in \mathbf{J}_{i} \cap \mathbf{J}_{d}}\left\lfloor\frac{T W_{i j}}{\bar{\tau}_{i j}}\right\rfloor, \max _{j \in \mathbf{J}_{i} \cap \mathbf{J}_{d}}\left(\min _{k \in \mathbf{K}_{i}^{-}}\left\lfloor\frac{\bar{\omega}_{k}}{\rho_{i k}^{\min } \beta_{j}^{\min }}\right\rfloor\right)\right\}$

Finally, an extension of these time-based concepts is introduced by allowing the earliest start time and shortest tail to be variables. In particular, new variables $\bar{E}_{i j}$ and $\overline{S T}_{i j}$ are introduced and bounded by their parameter counterparts as expressed by equation (42). Tightening constraints involving these variables are presented in section 3.3.3.

$\bar{E}_{i j} \geq \bar{\varepsilon}_{i j} ; \overline{S T}_{i j} \geq \bar{\sigma}_{i j} \forall i, j \in \mathbf{J}_{i}$

The next sections present a description of the algorithms and the constraints required to implement these methods. Section 3.3.2 presents the algorithms to (1) determine $\bar{\varepsilon}_{i j}$ and $\bar{\sigma}_{i j}$, (2) calculate $\bar{\mu}_{i}$ and $\bar{\omega}_{k}$, (3) identify groups of dependent tasks, and (4) define the convex hull of the points defining a feasible region of combinations of number of batches within a given subgroup. Finally, section 3.3.3 presents tightening constraints derived using these parameters.

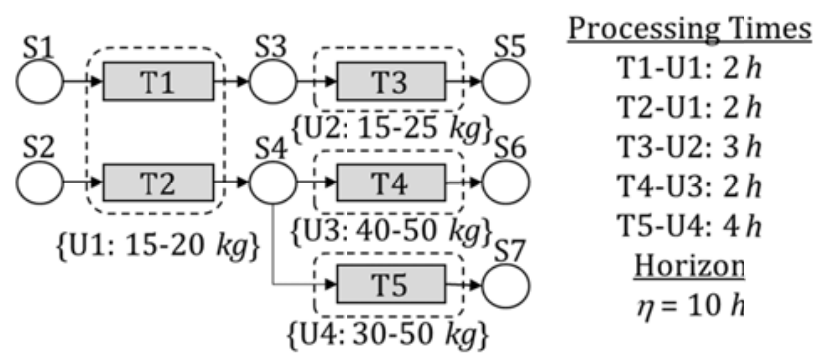

Figure 10. Groups based on task dependencies

$\mathrm{T} 1$ and $\mathrm{T} 2$ are trivially grouped because they are processed in the same unit. Tasks T4 and T5 are dependent and constitute a group because they share an input material. Tasks T3 and T4 can also be grouped because they consume materials produced by the same unit. Once a constraint relating the number of batches of the subgroup $\{\mathrm{T} 1, \mathrm{~T} 2\}$ is developed, it is propagated to the subgroup $\{\mathrm{T} 3, \mathrm{~T} 4\}$ to further tighten the formulation derived from the forward-propagation algorithm. 


\subsubsection{Algorithms}

The time- and inventory-based methods comprise four algorithms to determine as many families of parameters which are used to define tightening constraints. The algorithms in pseudo-code form can be found in the Appendix.

Earliest Start Time Algorithm: The basic idea of this algorithm is to find the first point $t$ on the time grid for which the maximum amount of a specific material that can be produced up to time $t$ exceeds the minimum amount required to produce one batch of the task whose earliest starting time is being calculated. The stair-shaped line in Figure 8(b) represents the production profile for the material if it uses all available resources and the dotted line is the minimum amount required by the task; their intersection defines the earliest starting time for the task. The algorithm uses three intermediate parameters to account for (1) the maximum amount of a material available at time $t$, (2) the cumulative amount of material a task can process in a specific unit starting at time $t$, and (3) the cumulative amount of material a task can process in all compatible units. The shortest tail algorithm is based on a modified shortest path procedure that is omitted here. The output of the algorithm is the set of parameters $\left\{\bar{\varepsilon}_{i j}\right\}_{i, j \in \mathbf{I}_{j}}$ and $\left\{\bar{\sigma}_{i j}\right\}_{i, j \in \mathbf{I}_{j}}$.

Forward-Propagation Algorithm: The objective of this algorithm is to calculate the maximum feasible amount that every task can produce within its time window and the corresponding maximum amounts of each material that can be produced within the given horizon. It employs equations 36-40 by mirroring its backward-propagation counterpart ${ }^{3}$, but considering not only material amounts, but also time restrictions. The algorithm detects if a task is limited by inventory (i.e. there is excess time in its window, but no additional input materials can be produced) or time (i.e. there is enough material to continue a task execution, but the time window is exceeded). The main output of the algorithm is the set of parameters $\left\{\bar{\mu}_{i}^{1}\right\}_{i \in \mathbf{I}^{*}}$

Group identification algorithm: The main goal of this algorithm is to define groups of tasks that are interrelated and whose production is limited by one another. The dependence between different tasks is introduced in three different ways: (1) tasks that share a processing unit, (2) tasks that share an upstream material, and (3) tasks that share an upstream unit. The first type of dependence is the simplest to analyze, since the time window for the common unit bounds the maximum combined amount its compatible tasks can process. The second and third types also define implicit upper bounds on the combined processing amounts, but considering the effect of limited upstream resources for every task. The output of this algorithm is the set of groups $d \in \mathbf{D}$

Feasible region's convex hull algorithm: This algorithm uses the groups previously identified to explicitly calculate the upper bounds on the production of each task within a given group. These upper bounds are not individual values for each task, but rather linear combinations of the number of batches for the tasks within a group. The algorithm begins with the calculation of the maximum number of batches for each task in a group and then generates the grid of feasible combinations of batches for all the tasks in the same group. Subsequently, the qhull algorithm 66 is used to predict the set $h \in \mathbf{H}_{d}$, for the number of linear constraints that define the convex hull of a feasible region for a particular subgroup $d$ and the correspondent coefficients of those constraints $\varphi_{i d h}$ and $\bar{\varphi}_{d h}$. These inequalities become tightening constraints as shown in the next section. 


\subsubsection{Constraints}

The parameters calculated in the previous algorithms are used to generate different sets of tightening constraints.

Eq. 43 is used to upper-bound the total time spent by a task in a single unit by using the time window defined through the Earliest Start Time Algorithm and equation 34. Eq. 44 is the equivalent constraint for a specific unit, using the time window defined in eq. 35 .

$\sum_{t} \tau_{i j} X_{i j t} \leq\left\lfloor\bar{\theta}_{i j} / \delta\right\rfloor \quad \forall i, j \in J_{i}$

$\sum_{t, i \in I_{j}} \tau_{i j} X_{i j t} \leq\left\lfloor\bar{\theta}_{i} / \delta\right\rfloor \quad \forall j$

The total cumulative production for a given task can be bounded above by $\bar{\mu}_{i}^{1}$ calculated in the Forward-Propagation Algorithm, according to equation (45).

$\sum_{j \in \mathbf{J}_{i}, t} B_{i j t} \leq \bar{\mu}_{i}^{1} \quad \forall i$

The parameters calculated using the Feasible Region's Convex Hull Algorithm can be used to bound a linear combination of the number of batches for tasks belonging to a common subgroup:

$\sum_{i \in \mathbf{I}_{d}, j \in \mathbf{J}_{i}, t} \varphi_{i d h} X_{i j t} \leq \bar{\varphi}_{d h} \quad \forall d, h \in \mathbf{H}_{d}$

Finally, equations 47-48 are used to further tighten the formulation when the parameters for earliest start time and shortest tail are allowed to vary. They express the relation between EST and ST variables for subsequent tasks.

$$
\begin{aligned}
& \bar{E}_{i j} \geq \bar{E}_{i^{\prime} j^{\prime}}+\tau_{i^{\prime} j^{\prime}}\left\lceil\frac{\rho_{i k}^{\min } \beta_{j}^{\min }}{\rho_{i^{\prime} k}^{\max } \beta_{j^{\prime}}^{\max }}\right\rceil \begin{array}{c}
\forall i, i^{\prime} \\
\forall j \in \mathbf{J}_{i}, j^{\prime} \in \mathbf{J}_{i^{\prime}} \\
\forall k \in \mathbf{K}_{i}^{-} \cap \mathbf{K}_{i^{\prime}}^{+}:\left|\mathbf{I}_{k}^{+}\right|=1
\end{array} \\
& \forall i, i^{\prime} \\
& \overline{S T}_{i^{\prime} j^{\prime}} \geq \overline{S T}_{i j}+\tau_{i j} \quad \forall j \in \mathbf{J}_{i}, j^{\prime} \in \mathbf{J}_{i \prime} \\
& \forall k \in \mathbf{K}_{i}^{-} \cap \mathbf{K}_{i^{\prime}}^{+}:\left|\mathbf{I}_{k}^{+}\right|=1
\end{aligned}
$$

\subsection{Reformulation}

The reformulation from Velez and Maravelias ${ }^{5}$ remains unchanged based on the extensions presented here. The reformulation uses a new integer variable, $N_{i j}$, that gives the number of times that task $i$ runs in unit $j$.

$\sum_{t} X_{i j t}=N_{i j} \quad \forall i, j \in \mathbf{J}_{i}$

$N_{i j}$ is bounded by the number of times a task can run during the horizon, $\eta$.

$0 \leq N_{i j} \leq\left\lfloor\eta / \tau_{i j}\right\rfloor \forall i, j \in \mathbf{J}_{i}$

Branching on $X_{i j t}$ leads equivalent schedules that have the total number of batches and the same objective, but with the start time of tasks shifted (Figure 11a). Branching on this new integer variable quickly finds schedules with different numbers of batches and different objectives (Figure 11b). 


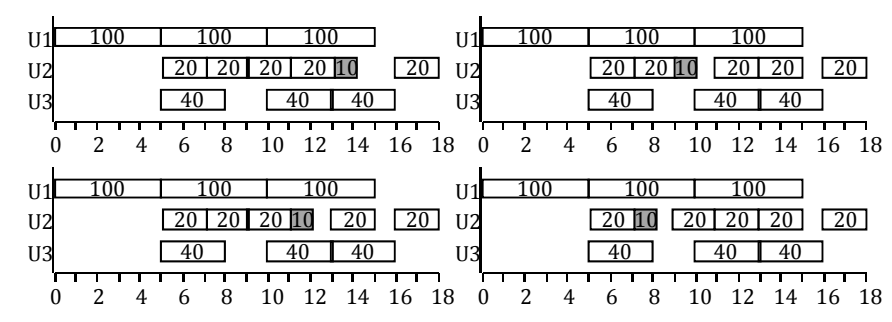

(a) Four equivalent schedules

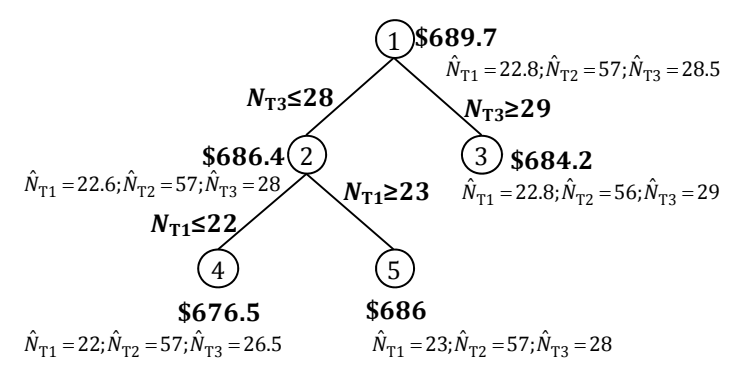

(b) Branching on $N_{i j}$

Figure 11. (a) Four equivalent schedules formed by shifting tasks earlier or later. The gray box represents an $X_{i j t}$ of 0.5 . All of these schedules have the same objective. (b) Results for branching on $N_{i j}$. The bound on the maximum profit (in bold) improves quickly.

In this paper, we introduce a similar reformulation for changeovers, where $N_{j}^{C O}$, is the number of changeovers from family in unit $j$.

$N_{j}^{C O}=\sum_{p p, t} Z_{p p} j t \quad \forall j$

$N_{j}^{C O}=\sum_{p, t} Z_{p j t}^{1} \quad \forall j$

\section{Examples}

We solve three large problems using the basic discrete-time formulation ${ }^{34}$ and the methods described in sections 2-3. We consider 4 or 12 formulations depending on whether there are changeovers. Formulations are named as FT.R.C, where T stands for the tightening method used (0 $=$ no tightening constraints, 1 = tightening constraints $29-32$ or 43-46), $\mathrm{R}$ stands for the reformulation $(0=$ no additional constraints/variables, $1=$ includes constraints $49-50$, and $2=$ includes constraints 49-52), and $\mathrm{C}$ stands for the changeover formulation $(1=$ changeover

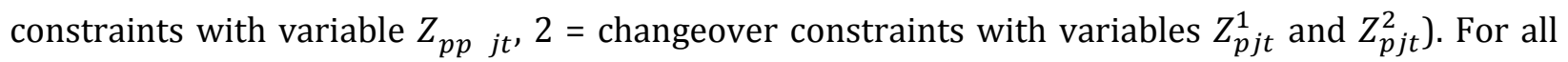
examples the multi-grid model from section 3.1 results in a single uniform grid. Since the main objective in industrial applications is to obtain very good (although maybe suboptimal) feasible solutions and the methods described in the previous sections are most effective in moving the bound on the solution, we also developed different options files to focus on finding good solutions. Option file 1 uses the default settings and option file 2 is set to use heuristics aggressively and to find good integer solutions (mipemphasis $=4$; fpheur $=1$, lbheur $=1$, rinsheur $=1$ in CPLEX). Note that customizing modern MIP solvers can by itself lead to significant improvements but it is beyond the scope of this paper. Instances in sections 4.2 .1 and 4.3 are solved using GAMS 23.9.2/CPLEX 12.4 on a computer with $6 \mathrm{~GB}$ of RAM and a $2.67 \mathrm{GHz}$ Intel Core (i7-920) processor running on Windows 7. Instances in sections 4.1 and 4.2.2 are solved using GAMS 24.4.1/CPLEX 12.6 on a computer with $8 \mathrm{~GB}$ of RAM and a $2.8 \mathrm{GHz}$ Intel Core (i7-930) processor running on Windows 7. Additional data and model statistics can be found in the Supporting Information.

\subsection{Example 1}

The first network we consider is modified from Papageorgiou and Pantelides ${ }^{71}$ to include utility and setup times (Figure 12). The network consists of 19 tasks, 8 units, and 27 materials. Materials have no storage, unlimited storage, or limited storage. There are two resources: one resource has a 
variable cost and the other has a variable availability (Figure 13). Raw material deliveries and order due times occur every 24 hours (Figure 13). There are setup times that depend on the task and the unit and last for 0,1 , or 2 hours. We use a one-hour time step over a 120-hour horizon. The objective is to maximize profit while meeting customer demand.

$P R F=\sum_{k} \pi_{k} S_{k \eta}-\sum_{k, t} \pi_{k} \xi_{k t}-\sum_{i, j \in \mathbf{J}_{i}, t} \alpha_{i j} X_{i j t}-\sum_{i, j \in \mathbf{J}_{i}, t} \alpha_{r t}^{r e s} U_{r t}$

where $\pi_{k}$ is the selling price of material $k, \alpha_{i j}$ is the fixed cost to process task $i$ in unit $j$, and $\alpha_{r t}^{r e s}$ is the cost to use one unit of resource $r$ during time period $t$. The model consists of constraints 1-4, 24, and 53.
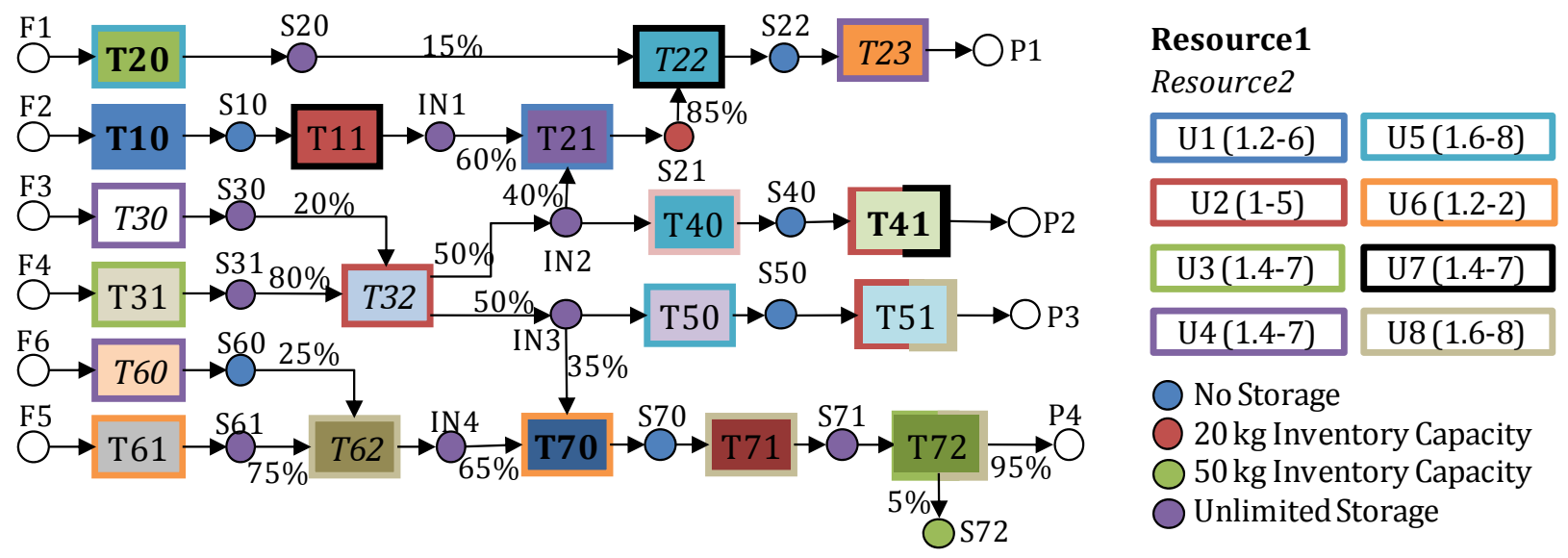

Figure 12. STN representation of Example 1. Task outline colors show the task-unit compatibility. Material colors indicate the storage policy. Fonts for the task name indicate which resource (if any) is used.

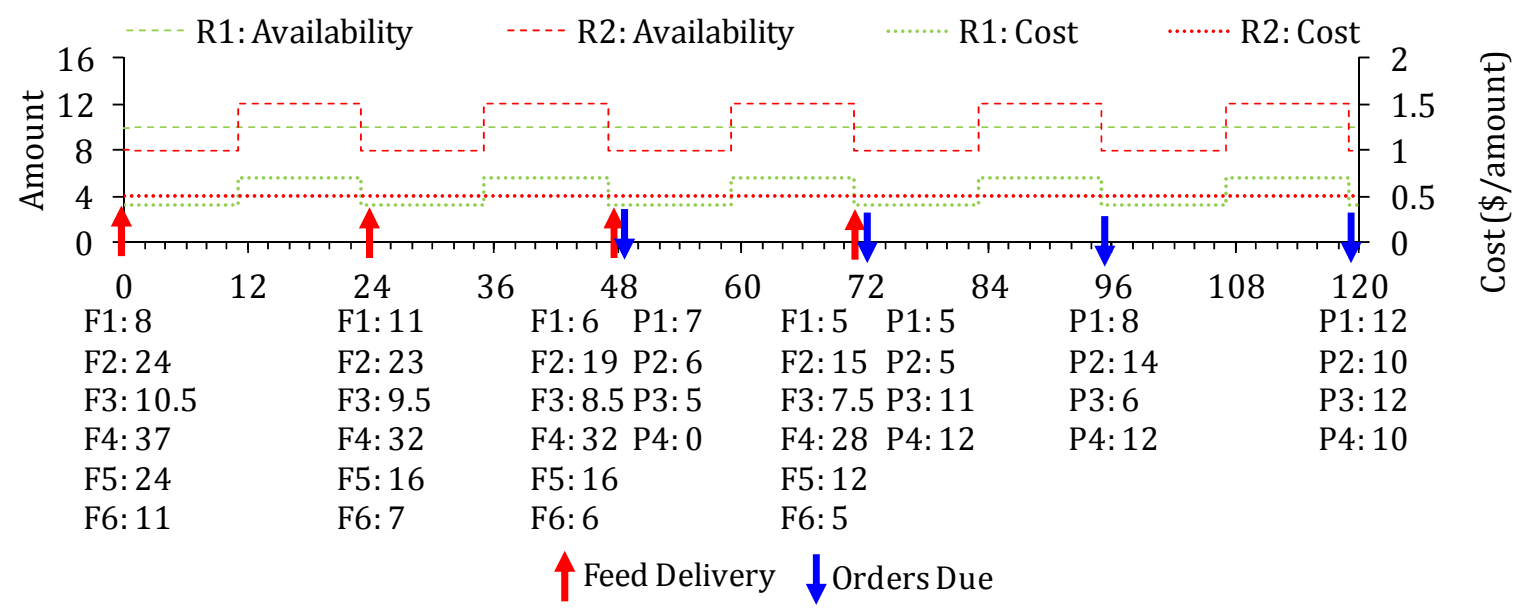

Figure 13. Problem data for Example 1, including utility availability and cost, raw material deliveries, and product orders.

Using the combined tightening and reformulation methods that define formulation F1.1, an objective value of $11,878.52$ was found with an optimality gap of $0.71 \%$ after one hour when option set 1 was used. If the option file is changed to option 2 to emphasize feasibility, an objective of $11,909.25$ was found with a gap of $0.72 \%$ after one hour. Overall, the best solution was found using 
the reformulation and the second options file (solution is shown in Figure 14), but the smallest gap was obtained using the reformulation and the first options file. In general, it is expected that an instance-specific set of options can lead to even better solutions though the identification of such options would require extensive testing.

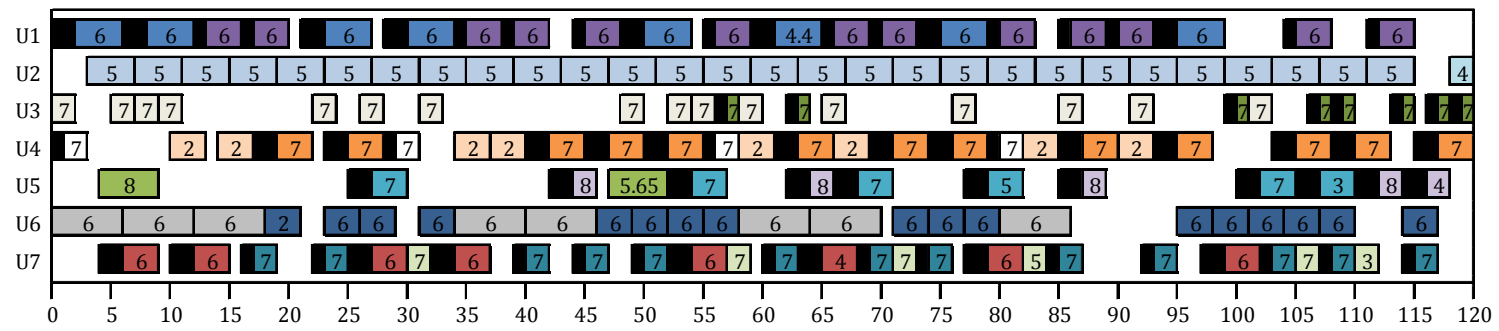

(a) Gantt Chart

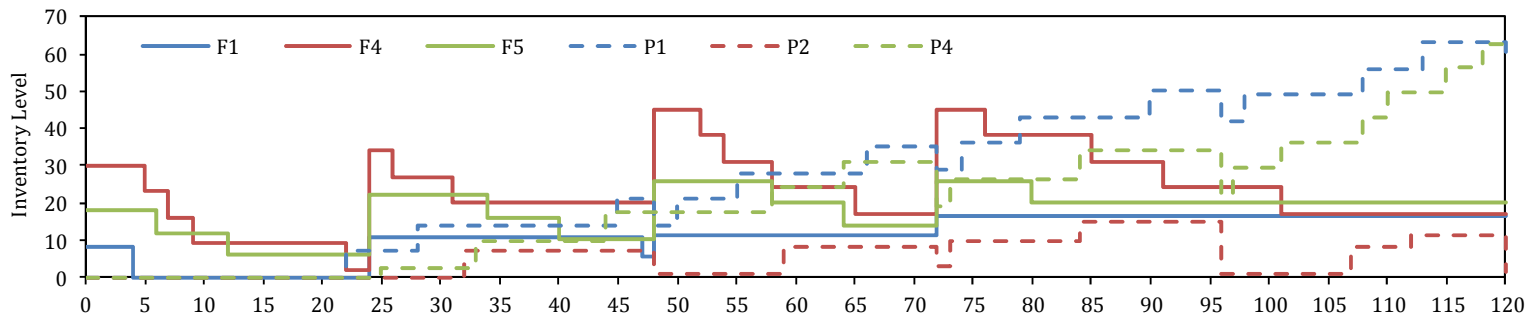

(b) Inventory Profiles

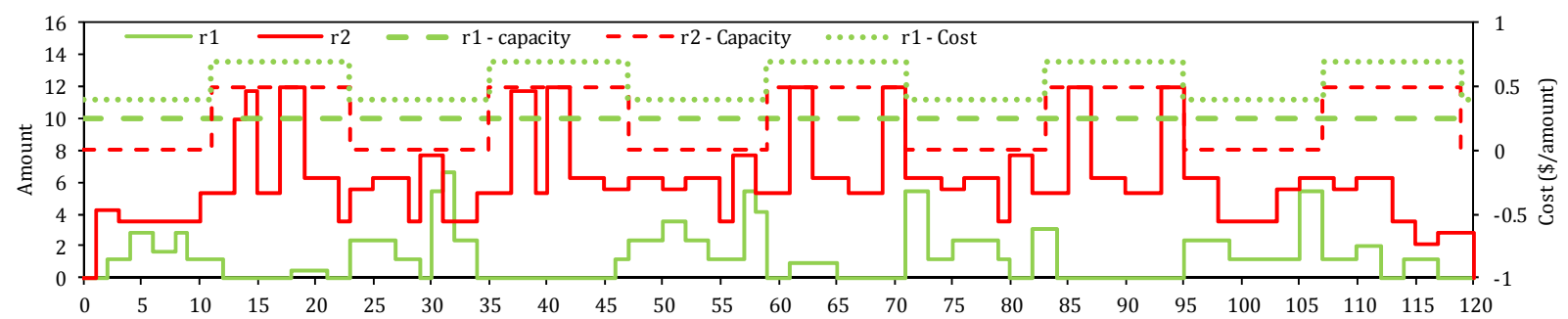

(c) Resource Profiles

Figure 14. Solution for Example 1. (a) Gantt chart for the optimal schedule. Setup times are shown in black. Colors indicate the task and match the task colors in the network shown in Figure 12. Batch sizes are given. (b) Inventory profiles for select feeds and products. (c) Resource cost, availability, and consumption during the time horizon.

\subsection{Kallrath Example}

The Kallrath network ${ }^{72}$ (Figure 15) is a benchmark problem that has been used in the literature as a challenge problem to test the effectiveness of different formulations and solution methods. It consists of batch tasks and does not have storage in units, buffer tanks, or changeovers. Task T2 has a variable conversion coefficient, where $20-70 \%$ of a batch is converted to S21 and the remainder is converted to S22. We consider two different problems, based on different objectives: makespan minimization and profit maximization.

\subsubsection{Makespan minimization}

The objective is to minimize the makespan as defined in equation 54 while meeting the given demand.

$M S \geq \sum_{i \in \mathbf{I}_{j}}\left(t+\tau_{i j}\right) X_{i j t} \quad \forall j, t$ 
The original formulation consists of constraints $1,2,4-7$, and 54 . We solve and present results for three instances that differ in the demand amounts using a time horizon of 60 hours. P0 is the original instance from Kallrath ${ }^{72}$, and P9 and P11 are two of the hardest instances that have been reported. Since this example does not include changeovers, we only consider the variations for the tightening methods and reformulation. Table 1 summarizes the results.

We compare our results to four previous continuous-time formulations:

(i) Wang and Guignard 73 (W\&G) used GAMS 2.5 with CPLEX 7.0

(ii) Janak and Floudas ${ }^{47}$ (J\&F) used an HP-UX/9000 with dual 440MHz CPUs with CPLEX 7.5

(iii)Vooradi, Shaik, and Gupta ${ }^{74}$ (VS\&G) used CPLEX 11.0 solver in GAMS 22.6 on $2.66 \mathrm{GHz}$ Intel Core 2 Duo Processor with 3 GB RAM

(iv) Vooradi and Shaik ${ }^{75}$ (V\&S) used CPLEX 12.2 solver in GAMS 23.5 on $2.66 \mathrm{GHz}$ Intel Core 2 Duo Processor with 3 GB RAM

\section{(v)}

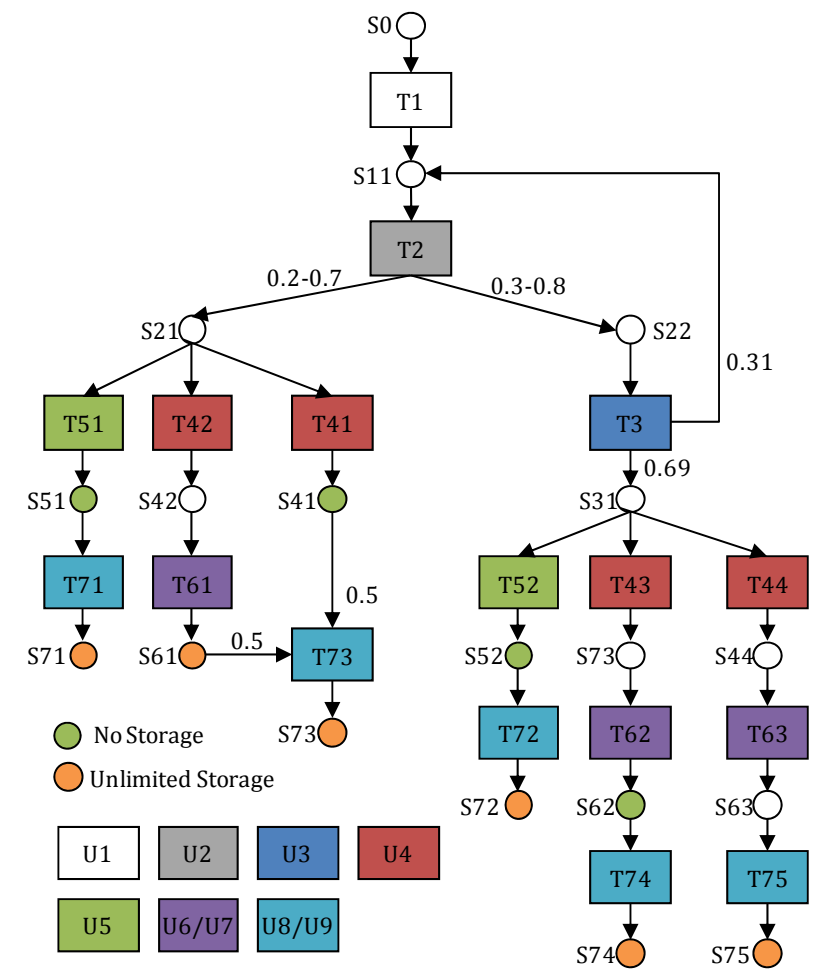

Figure 15. STN representation of the Kallrath example (Kallrath, 2002).

Table 1. Computational times in seconds and objective value (in parentheses) for the proposed formulation with tightening and reformulation and the three solution methods previously reported in the literature.

\begin{tabular}{|c|c|c|c|c|c|c|}
\hline & F1.1 & W\&G & $\mathrm{J} \& \mathrm{~F}$ & VS\&G & V\&S & \\
\hline P0 & $526.4(52)$ & $(56)^{1}$ & $(54)^{2}$ & & $40,000^{4}(52)$ & \\
\hline P9 & $256.8(32)$ & $217.2(36)$ & $983.7(34)$ & $16.1(34)$ & $40,000^{5}(33)$ & \\
\hline P11 & $1008.3(39)$ & & $36,000^{3}(40)$ & $80,000^{3}(40)$ & $40,000^{6}(40)$ & \\
\hline \multicolumn{7}{|c|}{${ }^{1}$ Total solution time not given, From Wang and Guignard, 2006} \\
\hline \multicolumn{7}{|c|}{ ²Solution found manually } \\
\hline \multicolumn{7}{|c|}{${ }^{3}$ Reached CPU resource limit } \\
\hline \multirow{2}{*}{\multicolumn{7}{|c|}{$\begin{array}{l}{ }^{4} \text { Reached CPU resource limit with } 7.6 \% \text { gap. Used a different computer with } 3.33 \mathrm{GHz} \text { and } 12 \mathrm{~GB} \text { RAM } \\
\text { 5Reached CPU resource limit with 3.3\% gap }\end{array}$}} \\
\hline & & & & & & \\
\hline \multicolumn{7}{|c|}{${ }^{6}$ Reached CPU resource limit with $4.52 \%$ gap } \\
\hline
\end{tabular}



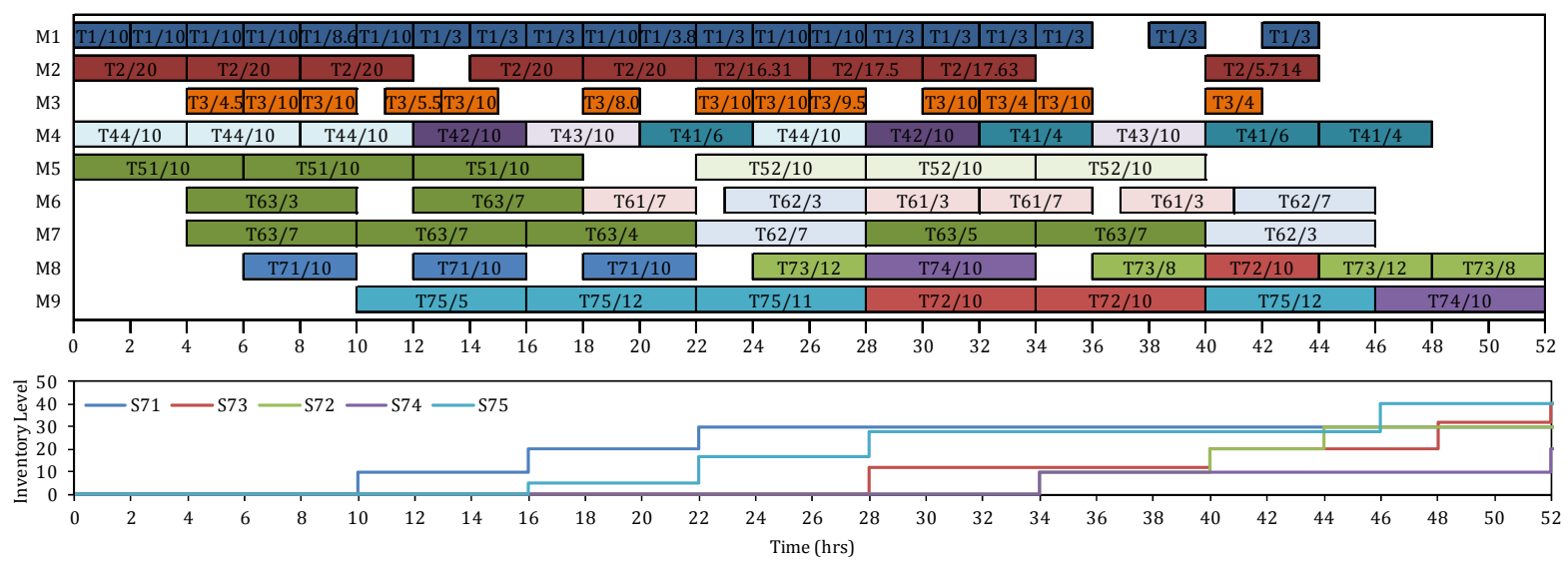

Figure 16. Optimal solution and product inventory levels for P0 of the Kallrath example.

All the proposed formulations lead to better results than previous approaches in the literature. They yield better solutions (e.g. P0, P11 with F1.1 compared to the rest) or are solved to optimality faster (e.g. P0 with F1.1 v. V\&S). Specifically, for P9 and P11, we find a better solution than previously reported in the literature and have much faster solution times for P0 and P11. In other words, the proposed methods allow us to solve problems that were open, i.e., the optimal solution was not known. Note that we observe order-of-magnitude improvements, so only a fraction of the difference in solution times might be attributed to the different hardware and software; most of the improvement is due to the application of the proposed methods.

Since we use a discrete-time model that satisfies the multi-grid conditions in section 3 , the optimal solution for our formulation is guaranteed to be the best possible solution. W\&G and J\&F use continuous-time models and declare optimality by resolving the problem using more points/periods until the objective does not change. V\&S also use a continuous-time model and use the same number of event points found in J\&F. Unlike the discrete-time model, this solution method does not guarantee that the best possible schedule is feasible for the formulation. For example, for instance P0, the original problem proposed by Kallrath and the most difficult, most other methods have not been able to find good solutions. With the proposed methods, P0 is solved to optimality in less than 10 minutes (solution shown in Figure 16). For P9, W\&G and J\&F report that the problem has been solved to optimality, but we were able to find a better solution with a makespan $5 \%$ shorter. With a discrete-time model and the solutions methods presented here, the optimal solution was found in less than 5 minutes. Again, problem P11 is not solved to optimality by the previous formulations within over 20 hours. With the discrete-time model and proposed methods, the optimal solution is found in less than 30 minutes, over an order-of-magnitude improvement.

\subsubsection{Profit maximization}

In this subsection, we illustrate the time- and inventory-based methods described in section 3.3. We consider again the process depicted in Figure 15 with the same data in terms of variable conversion coefficients, unit/storage capacities and demands, but now we change our focus to profit maximization. Table 2 summarizes the results for problem P0 as described before and a horizon of 60 hours. The original formulation is able to find the optimal solution, but it fails to prove optimality after one hour. When the tightening and reformulations methods (eqs. 44-46 and 49-50) 
are added (formulation F1.1), optimality is proven in about 12 min. Unlike makespan minimization, there are no available data in the literature to compare for profit maximization. Figure 17 shows the resulting Gantt chart. Additional data and model statistics can be found in the Supporting Information.

\subsection{Dow Example}

The Dow example (Nie et al.76) consists of five main product lines, A-E (Figure 18). Each product must go through exactly the same six steps. Step 1 is a batch process; two identical units (units UB1 and UB2) are available for this step, and these units can also be used to store their output material $\left(\{\mathrm{UB} 1, \mathrm{UB} 2\} \in \mathbf{J}^{S}\right.$ ). Steps 2 (unit UT1), 3 (unit UT2), and 5 (unit UT3) are buffer tanks $\left(\{\mathrm{UT1}\right.$, UT2, UT3 $\} \in \mathbf{J}^{B}$ ). Steps 4 (unit UC1) and 6 (unit UC2) are continuous processes (\{UC1, UC2 $\} \in$ $\mathbf{J}^{C}$ ). A sixth product, $\mathrm{F}$, is required as input to the batch processing tasks for product lines A-C. Product $\mathrm{F}$ is produced by running just the batch processes (step 1). In the continuous processing units, material cannot be stored, and there are changeovers between product lines of different families (A, C, and D are in the same family, and B and E are each in their own families). Also, the capacity of continuous processing unit UC2 is reduced every time it operates; the lost capacity is equal to the batch size processed. A replenishing task must be run to restore the capacity when it falls too low and can only be run when the capacity falls below $25 \%$ of its maximum. To create the STN for this process, we introduce new materials and tasks for each product line and for each step (Figure 19). The capacity of unit UC2 is modeled as a material, CAP, which is consumed every time a task runs in the unit; when the inventory of CAP is low, the task Replen is run to produce more. We set the threshold for the replenishing task by setting its minimum batch size to $75 \%$ of the storage capacity for material CAP: $\beta_{\text {Replen,UC2 }}^{\min }=0.75 \gamma_{C A P}$. To ensure the capacity is completely refilled, we use eq. 55.

$B_{\text {Replen,UC2,t }}+S_{C A P, t} \geq \gamma_{C A P} X_{\text {Replen,UC2,t }}$

There are no changeovers between task Replen and any of the other tasks in UC2. Demand must be met exactly as expressed in eq. 56, where we replace the parameter $\xi_{k t}$ with the negative variable $D_{k t}$. There is a penalty for violating the safety stock at the end of the horizon (eq. 57). The objective is to maximize profit over three days (with a 1-hour time step) (eq. 58). The revenue generated from selling final products depends on when the products are delivered. Costs are incurred for changeovers, inventory, and safety stock violations at the end of the horizon. 
Table 2. Objective value and CPU time for the formulations F0.0 and F1.1.

\begin{tabular}{cccccc} 
& \multirow{2}{*}{ Objective } & \multicolumn{3}{c}{ CPU time (s) } & \multirow{2}{*}{ Total } \\
\hline F0.0 & 4599.9 & 0.00 & 3600 & 3600 & $<0.01$ \\
F1.1 & 4599.9 & 121.73 & 565.11 & 686.84 & 0.00 \\
\hline
\end{tabular}

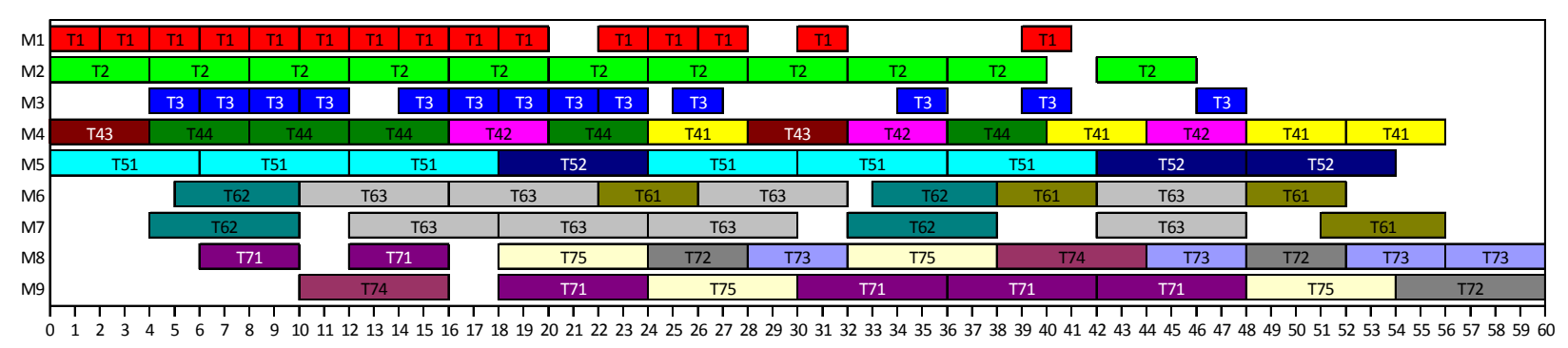

Figure 17. Gantt chart for the optimal solution for $\mathrm{P} 0$ of the Kallrath example with profit maximization.

$$
\begin{aligned}
& \sum_{\varepsilon_{o} \leq t \leq \lambda_{o}}-D_{k t}=\xi_{k o} \quad \forall o, k \in \mathbf{K}_{o} \\
& \gamma_{k}^{\text {safety }}-S_{k}^{v i o l} \leq S_{k \eta} \quad \forall k \\
& P R F=-\sum_{k, t} \pi_{k t} D_{k t}-\sum_{k, t} \alpha_{k t}^{i n v} S_{k t}-\sum_{p, p \neq p, j, t} \alpha_{j}^{C o} Z_{p p} j t-\sum_{k} \alpha_{k}^{v i o l} S_{k}^{v i o l}
\end{aligned}
$$

where $\mathbf{K}_{o}$ is the material due in order $o, \xi_{k o}$ is the amount of material $k$ required for order $o, \varepsilon_{o} / \lambda_{o}$ is the earliest/latest delivery time for order $o, \gamma_{k}^{\text {safety }}$ is the safety stock level for material $k$, $S_{k}^{v i o l} \geq 0$ is the safety stock violation for material $k$ at the end of the horizon, and $\alpha_{k t}^{i n v} / \alpha_{j}^{C O} / \alpha_{k}^{v i o l}$ is the inventory/changeover/violation cost.

The model for this process consists of constraints 2, 4, 16, 19-23, and 55-58 along with 8-11 or 9 plus 12-15 to model changeovers (if changeover constraints 9 plus 12-15 are used we replace $Z_{p p^{\prime} j t}$ with $Z_{p j t}^{1}$ and remove the sum over $p^{\prime}$ in eq. 58). Note that all tasks occurring in units UT1, UT2, UT3, UC1, and UC2 are included in the set $\mathbf{I}^{C}$. To model the changeovers, tasks in product lines A, $\mathrm{C}$, and D and occurring in UC1 or UC2 belong to F1; tasks in product line B and occurring in UC1 or UC2 belong to F2; and tasks in product line E and occurring in UC1 or UC2 belong to F3.

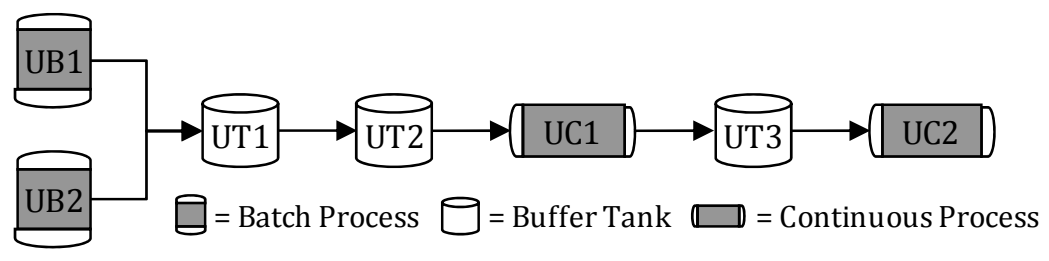

Figure 18. Equipment layout of the Dow example 


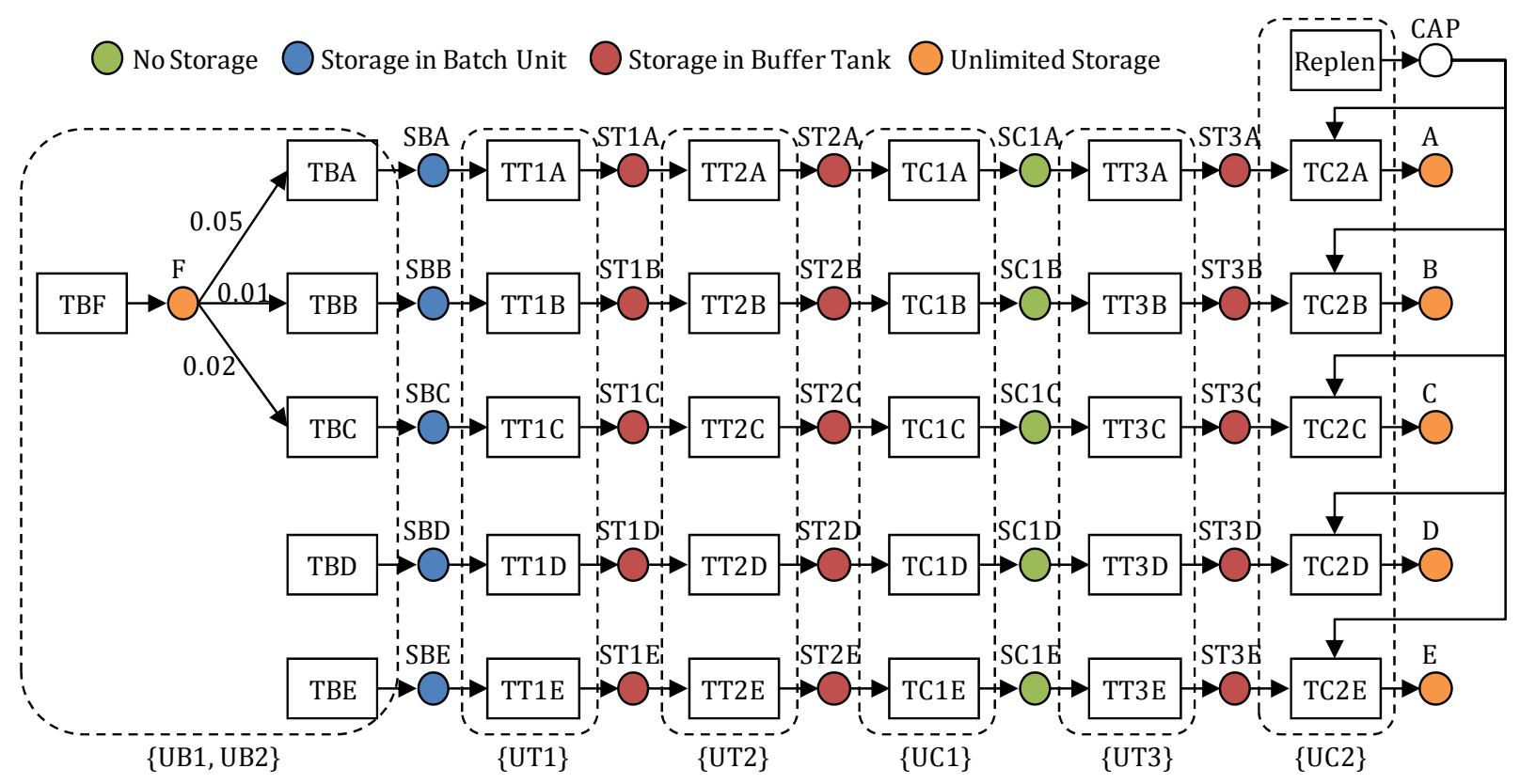

Figure 19. STN representation of the Dow example. Dashed boxes show the unit compatibility.

We consider maximizing profit after 72 hours using all the formulations and the second set of options file described at the beginning of this section. The best results are obtained when the changeover formulation 1 is used. We observed that adding only reformulations improves the optimality gap, whereas adding the tightening constraints makes it more difficult to find integer solutions. Reformulation 2 has the smallest gap and best solutions. Options file 2 finds good solutions with less than a 5\% gap for nearly all formulations. The best solution found is shown in Figure 20. Additional data and model statistics can be found in the Supporting Information.

\section{Conclusions}

The goal of this paper was to illustrate how advances in MIP models and solution methods allow us to address large-scale problems with a range of complex processing constraints that are common in real-world applications. First, we showed that discrete-time models can be easily extended to account for, among others, variable conversion coefficients, setups and changeovers, storage in processing units and buffer tanks, intermediate release and due times, and time-varying resource availability and pricing. Second, we showed how tightening methods and reformulations are applicable to these problems, leading to (near) optimal solutions in time frames that are practically relevant (less that one hour). The proposed methods allow us to solve to optimality instances for which the optimal solutions were not known. Most importantly, the proposed methods allow us to address large-scale problems that until recently were thought to be intractable.

\section{Acknowledgements}

The authors thank Yisu Nie, Lorenz T. Biegler, John Wassick, and Carlos Villa for providing the data for the Dow example (subsection 4.3). The authors acknowledge financial support from the 
National Science Foundation under Grant CBET-1066206, and from the American Chemical Society

- Petroleum Research Fund under Grant PRF-53313ND9. 


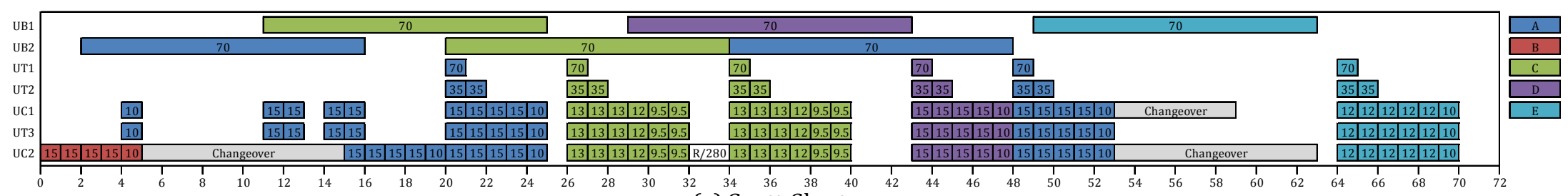

(a) Gantt Chart

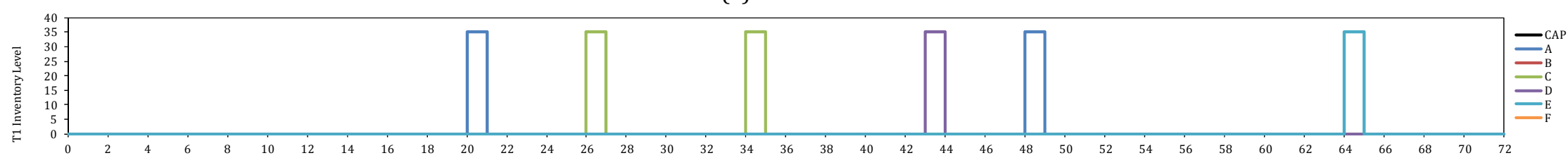

(b) Inventory Profiles for Buffer Tank T1

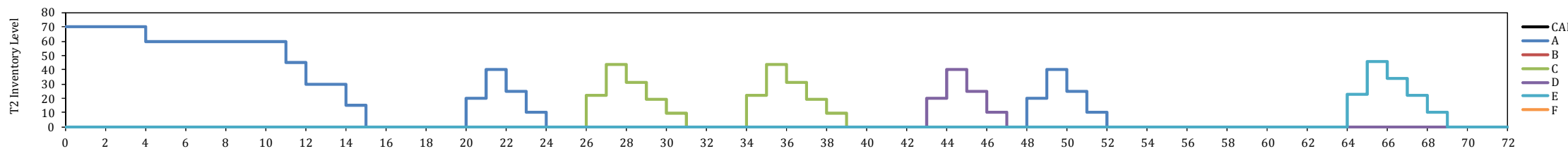

(c) Inventory Profiles for Buffer Tank T2

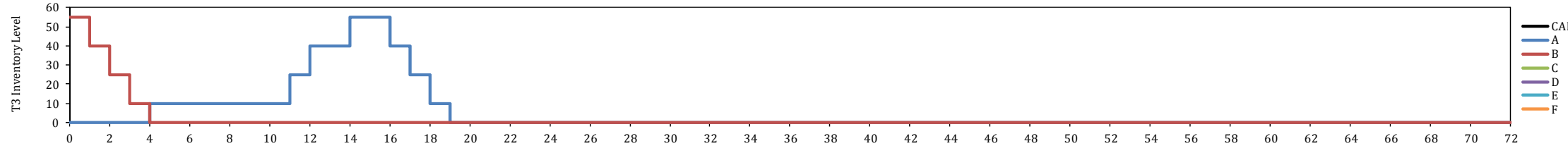

(d) Inventory Profiles for Buffer Tank T3

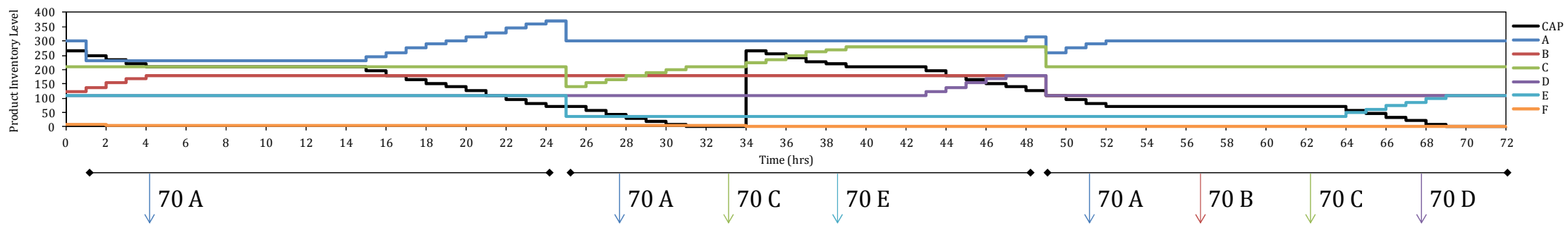

(e) Product Inventory Levels and Amounts Delivered

Figure 20. (a)Gantt chart and inventory profiles for (b) buffer tank T1, (c) buffer tank T2, (d) buffer tank T3, and (e) final products for the best solution found for the Dow example. Arrows show the amount of product that must be delivered in the time window shown by the black horizontal lines. 


\section{Notation}

Indices

$d \in \mathbf{D} \quad$ Groups of dependent tasks

$h \in \mathbf{H}_{d} \quad$ Convex hull constraints for inventory- and time-based algorithms

$i \in \mathbf{I} \quad$ Tasks

$j \in \mathbf{J} \quad$ Units

$k \in \mathbf{K} \quad$ Materials

$m \in \mathbf{M} \quad$ Combinations of number of batches to study attainable production regions.

$o \in \mathbf{0} \quad$ Orders

$p \in \mathbf{P} \quad$ Product families

$t \in \mathbf{T} \quad$ Time points

Sets

$\mathbf{I}_{k}^{+} / \mathbf{I}_{k}^{-} \quad$ Tasks producing/consuming material $k$

$\mathbf{I}_{j} \quad$ Tasks that can occur in unit $j$

$\mathbf{I}_{p} \quad$ Tasks belonging to family $p$

$\mathbf{I}^{C} \quad$ Continuous processes

I $^{O} \quad$ Tasks for which $\mu_{i}$ still needs to be calculated for algorithm in section 3.2.3.

$\mathbf{I}^{V} \quad$ Tasks that can produce materials with variable conversion coefficients

$\mathbf{J}^{\mathrm{CO}} \quad$ Units with changeovers

$\mathbf{J}^{B} \quad$ Buffer tanks

$\mathbf{J}^{S} \quad$ Processing units where storage is allowed

$\mathbf{J}_{i} \quad$ Units that can process task $i$

$\mathbf{J}_{k}^{S} \quad$ Processing units that can store material $k$

$\mathbf{K}_{i}^{+} / \mathbf{K}_{i}^{-} \quad$ Materials produced/consumed by task $i$

$\mathbf{K}^{F} \quad$ Final products

$\mathbf{K}_{j}^{S} \quad$ Materials that can be stored in unit $j$

$\mathbf{K}_{j}^{-} \quad$ Materials that can be consumed by unit $j$

$\mathbf{K}^{O} \quad$ Materials for which $\omega_{k}$ still needs to be calculated for algorithm in section 3.2.3.

$\mathbf{K}^{M T} \quad$ Materials that can be produced by multiple tasks

$\mathbf{P}_{j} \quad$ Families present in unit $j$

Parameters

$\alpha_{i j}$

$\alpha_{j}^{C O}$

$\alpha_{k}^{\text {viol }}$

$\alpha_{k t}^{i n v}$

$\alpha_{r t}^{r e s}$

$\beta_{i j}^{\min } / \beta_{i j}^{\max }$

$\beta_{j}^{\text {Smax }}$

$\gamma_{k}$

$\gamma_{k}^{\text {safety }}$

$\bar{\varepsilon}_{i j}$

$\bar{\varepsilon}_{j}$

$\varepsilon_{j}^{m}$

$\varepsilon_{o} / \lambda_{o}$

Processing cost for task $i$ in unit $j$

Changeover cost for unit $j$

Safety stock violation cost for material $k$

Inventory cost for material $k$ at time $t$

Cost to use one unit of resource $r$ during time period $t$

Minimum/maximum batch size of task $i$ in unit $j$

Maximum amount of material that can be stored in unit $j$

Maximum amount of material $k$ that can be stored

Safety stock level for material $k$

Earliest starting time for task $i$ in unit $j$

Earliest starting time for unit $j$

Number of batches in unit $j$ in combination $m$

Earliest/latest delivery time for order $o$ 


\begin{tabular}{|c|c|}
\hline$\eta$ & Horizon \\
\hline $\bar{\theta}_{i j}$ & Time window for task $i$ in unit $j$ \\
\hline $\bar{\theta}_{j}$ & Time window for unit $j$ \\
\hline$\theta_{r t}$ & Amount of resource $r$ available at time $t$ \\
\hline$\lambda_{i j}$ & Setup time for unit $j$ before processing task $i$ \\
\hline$\mu_{i}$ & Minimum amount task $i$ must produce to satisfy customer demand \\
\hline$\mu_{i}^{1}$ & $\begin{array}{l}\text { Smallest amount that can be produced by task } i \text { using any combination of units } \\
\text { whose minimum production is at least } \mu_{i}\end{array}$ \\
\hline$\mu_{i}^{2}$ & $\begin{array}{l}\text { Smallest value greater than } \mu_{i}^{1} \text { that is a linear, integer combination of the maximum } \\
\text { unit capacities }\end{array}$ \\
\hline $\bar{\mu}_{i}$ & Maximum amount that task $i$ can process within the given horizon \\
\hline $\bar{\mu}_{i}^{1}$ & $\begin{array}{l}\text { Largest amount that can be produced by task } i \text { using any combination of units } \\
\text { whose maximum production is at most } \bar{\mu}_{i}\end{array}$ \\
\hline$\xi_{k t}$ & $\begin{array}{l}\text { Amount of material } k \text { delivered at time } t(>0) \text { or due to customers at time } t(<0) ; \xi_{k 0} \\
\text { is the initial inventory of material } k \text {, including intermediates. }\end{array}$ \\
\hline$\xi_{k o}$ & Amount of material $k$ required for order $o$ \\
\hline$\pi_{k}$ & Selling price of material $k$. \\
\hline & Conversion coefficient of material $k$ produced $(>0)$ or consumed $(<0)$ by task $i$ \\
\hline$\rho_{i k}^{\min } / \rho_{i k}^{\max }$ & Minimum/maximum conversion coefficients of task $i$ to material $k$. \\
\hline$\sigma_{p p j}$ & Changeover time from family $p$ to family $p^{\prime}$ in unit $j$ \\
\hline $\bar{\tau}_{i j}$ & Processing time for task $i$ in unit $j$ \\
\hline$\tau_{i j k}^{R}$ & Residence time of material $k$ produced by task $i$ in unit $j$ \\
\hline$\tau_{i j}^{\min } / \tau_{i j}^{\max }$ & Minimum/maximum number of time periods continuous task $i$ can run in unit $j$ \\
\hline $\begin{array}{l}\varphi_{i d h} \\
\bar{\varphi}_{d h} \\
\psi_{i r} \\
\omega_{k} \\
\bar{\omega}_{k}\end{array}$ & $\begin{array}{l}\text { Coefficient for task } i \text { of group } d \text { in equation } h \\
\text { Right-hand-side coefficient for group } d \text { in equation } h \\
\text { Amount of resource } r \text { used per unit batch size of task } i \text { over one time period } \\
\text { Minimum amount of material } k \text { to satisfy customer demand } \\
\text { Maximum amount of material } k \text { that can be produced within the given horizon }\end{array}$ \\
\hline \multicolumn{2}{|c|}{ Continuous Variables } \\
\hline$B_{i j t}$ & Batch size of task $i$ processed in unit $j$ starting at time $t$ \\
\hline$B_{i j k t}^{V}$ & Amount of material $k$ produced by task $i$ in unit $j$ starting at time $t$ \\
\hline$D_{k t}$ & Amount of material $k$ delivered at time $t$ \\
\hline $\bar{E}_{i j}$ & Variable earliest start time for task $i$ in unit $j$ \\
\hline$F_{k j j t}$ & Flow of material $k$ from unit $j$ to $j^{\prime}$ at time $t$ \\
\hline$M S$ & Makespan \\
\hline$P R F$ & Profit \\
\hline$Q_{i}$ & Total amount that task $i$ processes (equations 27,37 ) \\
\hline$Q_{i k}^{V}$ & Total amount of material $k$ that task $i$ produces (equations 27,37 ) \\
\hline$R_{p j t}$ & One if unit $j$ is available to start a task belonging to family $p$ at time $t$ \\
\hline$U_{r t}$ & Amount of utility $r$ used at time $t$ \\
\hline$S_{k t}$ & Inventory of material $k$ at time $t$ \\
\hline$S_{k j t}^{P}$ & Amount of material $k$ being stored in processing unit $j$ at time $t$ \\
\hline$S_{k}^{v i o l}$ & Safety stock violation for material $k$ at the end of the horizon \\
\hline$\overline{S T}_{i j}$ & Variable shortest tail for task $i$ in unit $j$ \\
\hline
\end{tabular}


$X_{i j t}=1$ if unit $j$ processes task $i$ starting at time $t$

$Z_{p p j t} \quad=1$ if there is a changeover from family $p$ to family $p^{\prime}$ in unit $j$ at beginning at time $t$

$Z_{p j t}^{1} \quad=1$ if the changeover from family $p$ (to any other family) starts at time $t$

$Z_{p j t}^{2} \quad=1$ if the changeover to family $p$ (from any other family) ends at time $t$

Integer Variables

$N_{i j} \quad$ Number of times that task $i$ runs in unit $j$

$N_{j}^{C O} \quad$ Number of changeovers from family in unit $j$ 


\section{Appendix: Algorithms for Time- and Inventory-Based Methods}

\section{Earliest Start Time Algorithm}

The earliest start time algorithm uses the following parameters:

$\psi_{k t}^{E}: \quad$ Maximum amount of material $k$ available at time $t$

$\chi_{i j t}^{E}$ : Cumulative amount of material task $i$ can process in unit $j$ starting at or before time $t$

$\phi_{i t}^{E}$ : Cumulative amount of material task $i$ can process in any unit starting at or before time $t$ $E S T_{i j}$ : Earliest time task $i$ can begin in unit $j$

$$
\begin{array}{ll}
1 & \text { for } t=0 \text { to } \eta \\
2 & \psi_{k t}^{E}=\sum_{0 \leq t^{\prime} \leq t} \xi_{k t^{\prime}}+\sum_{i \in \mathbf{I}_{k}^{+}}\left[\rho_{i k} \min \left\{\sum_{j \in J_{i}} \chi_{i j\left(t-\tau_{i j}\right)}^{E}, \phi_{i\left(t-\tau_{i}^{m i n}\right)}^{E}\right\}\right] \\
3 & \chi_{i j t}^{E}=\min \left\{\min _{k \in \mathbf{K}_{i}^{-}}\left\{-\psi_{k t}^{E} / \rho_{i k}\right\}, \chi_{i j\left(t-\tau_{i j}\right)}^{E}+\beta_{j}^{\text {max }}\right\} \\
4 & \text { If } \chi_{i j t}^{E}<\beta_{j}^{\text {min }}, \chi_{i j t}^{E}=0 \\
5 & \phi_{i t}^{E}=\min \left\{\min _{k \in \mathbf{K}_{i}^{-}}\left\{-\psi_{k t}^{E} / \rho_{i k}\right\}, \sum_{j \in \mathbf{J}_{i}} \chi_{i j t}^{E}\right\} \\
6 & \text { end } \\
7 & E S T_{i j}=\min \left\{t: \chi_{i j t}^{E}>0\right\}
\end{array}
$$

This algorithm loops over all time points to determine the earliest time a task can start. $\psi_{k t}^{E}$ is equal to the total supply of material $k$ up to time $t$ plus the maximum amount that can be produced, which is the minimum of the amount produced in all units and the amount produced by all tasks. $\chi_{i j t}^{E}$ is initially set based on the amount of input material available for task $i$ (first term), but cannot grow by more than $\beta_{j}^{\max }$ every $\tau_{i j}$ time periods (second term). If $\chi_{i j t}^{E}$ is less than the minimum unit capacity, $\beta_{j}^{\text {min }}$, then no material can be processed by task $i$ in unit $j$ and $\chi_{i j t}^{E}=0$. The value of $\phi_{i t}^{E}$ is based on the total amount of input material available and is at most the sum of the amounts that can be produced in all units that process task $i$. Finally, the earliest start time is the earliest time for which $\chi_{i j t}^{E}$ is nonzero.

\section{Forward-Propagation Algorithm}

$$
\begin{array}{lc}
1 & \bar{\omega}_{k}=0 \forall k ; \bar{\mu}_{i}=0 \forall i ; \mathbf{K}^{O}=\mathbf{K} ; \mathbf{I}=\mathbf{I}^{O} \\
2 & \text { While }\left|\mathbf{K}^{O}\right|>0 \text { or }\left|\mathbf{I}^{O}\right|>0 \\
3 & \text { for } k \in \operatorname{argmin}\left\{\left|\mathbf{I}_{k}^{-} \cap \mathbf{I}^{O}\right|: k \in \mathbf{K}^{O}\right\} \\
4 & \mathbf{K}^{O}=\mathbf{K}^{O} \backslash\{k\} \\
5 & \text { Set } \bar{\omega}_{k} \text { from eq. } 39 \\
6 & \mathbf{I}^{O}=\mathbf{I}^{O} \backslash\{i\} ; \\
7 & \text { Set } \bar{\mu}_{i} \text { from eq. } 38 \\
8 & \text { Calculate } \bar{\mu}_{i}^{1} \text { from eq. } 40 \\
9 & \text { If } \exists k: \sum_{i \in \mathbf{I}_{k}^{-}} \rho_{i k}^{\max } \bar{\mu}_{i}^{1}>\bar{\omega}_{k} \\
10 & \text { Then } \mathbf{K}^{O}=\mathbf{K} ; \mathbf{I}^{O}=\mathbf{I}
\end{array}
$$

\section{Task dependence algorithm}

The task dependence algorithm uses the following additional sets: 
$\mathbf{J}_{k}^{+}\left(\mathbf{J}_{k}^{-}\right)=\left\{j \in \mathbf{J}_{i}: i \in \mathbf{I}_{k}^{+}\left(\mathbf{I}_{k}^{-}\right)\right\}$: Units that produce (consume) material $k$.

$\mathbf{K}_{j}^{+}\left(\mathbf{K}_{j}^{-}\right)=\left\{k \in \mathbf{I}_{k}^{+}\left(\mathbf{I}_{k}^{+}\right): i \in \mathbf{I}_{j}\right\}$ : Materials produced (consumed) by task $j$.

$\mathbf{I}_{d}$ : Tasks in the subgroup $d$.

1 Set $d=0$

Subgroups type I: Tasks sharing a unit

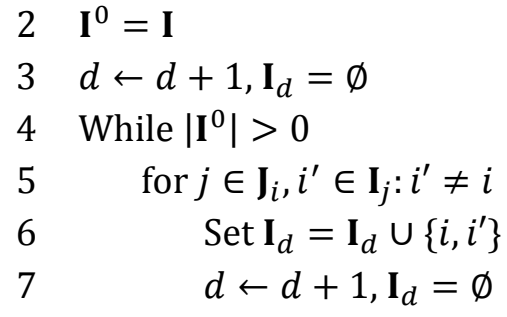

Subgroups type II: Tasks sharing common upstream material

8 While $\left|\mathbf{I}^{0}\right|>0$

9 for $k \in \mathbf{K}_{i}^{-}, i^{\prime} \in \mathbf{I}_{k^{\prime}}^{-}: i^{\prime} \neq i$

$10 \quad$ Set $\mathbf{I}_{d}=\mathbf{I}_{d} \cup\left\{i, i^{\prime}\right\}$

$11 \quad d \leftarrow d+1, \mathbf{I}_{d}=\varnothing$

Subgroups type III: Tasks sharing common upstream unit

$12 \mathbf{I}^{0}=\mathbf{I}$

13 While $\left|\mathbf{I}^{0}\right|>0$

14 for $k \in \mathbf{K}_{i}^{-}, j \in \mathbf{J}_{k}^{+}, k^{\prime} \in \mathbf{K}_{j}^{+}: k^{\prime} \neq k, i^{\prime} \in \mathbf{I}_{k^{\prime}}^{-}: i^{\prime} \neq i$

$15 \quad$ Set $\mathbf{I}_{d}=\mathbf{I}_{d} \cup\left\{i, i^{\prime}\right\}$

$16 \quad d \leftarrow d+1, \mathbf{I}_{d}=\emptyset$

$17 \mathbf{I}^{0}=\mathbf{I}$

Feasible region's convex hull algorithm

$1 \quad \mathbf{D}^{0}=\mathbf{D}$

2 While $\left|\mathbf{D}^{O}\right|>0$

3 If Type I then set $N_{i d}^{\max }$ from eq. 41

4 Otherwise

$5 \quad$ for $i \in \mathbf{I}_{d}$

$6 \quad$ Fix $\sum_{t, j \in \mathbf{J}_{i}} X_{i j t}$ to a value $N$ in $\left[0, N_{i d}^{\max }\right]$

7 Solve Shah model for feasibility

$8 \quad$ If infeasible then break and set $N_{i d}^{\max } \leftarrow N-1$

9 Generate feasible set of points $\mathbf{S}$ in $\left[0, N_{i d}^{\max }\right]^{\left|\mathbf{I}_{d}\right|}$

10 Calculate conv (S) using qhull (parameters $\varphi_{i d h}$ and $\bar{\varphi}_{d h}$ )

$11 \quad$ Set $\mathbf{D}^{0} \leftarrow \mathbf{D}^{0} \backslash\{d\}$ 


\section{References}

1. Velez, S.; Maravelias, C. T. Multiple and nonuniform time grids in discrete-time MIP models for chemical production scheduling. Comput Chem Eng 2013, 53, 70-85.

2. Velez, S.; Maravelias, C. T. Theoretical Framework for Formulating MIP Scheduling Models with Multiple and Non-Uniform Discrete-Time Grids. Comput Chem Eng 2014, (0).

3. Velez, S.; Sundaramoorthy, A.; Maravelias, C. T. Valid Inequalities Based on Demand Propagation for Chemical Production Scheduling MIP Models. AlChE J. 2013, 59 (3), 872887.

4. Merchan, A. F.; Maravelias, C. T. Preprocessing and Tightening Methods for MIP Chemical Production Scheduling Models. In preparation 2014.

5. Velez, S.; Maravelias, C. T. Reformulations and Branching Methods for Mixed-Integer Programming Chemical Production Scheduling Models. Ind. Eng. Chem. Res. 2013, 52 (10), 3832-3841.

6. Merchan, A. F.; Velez, S.; Maravelias, C. T. Tightening methods for continuous-time mixedinteger programming models for chemical production scheduling. AlChE J. 2013, 59 (12), 4461-4467.

7. Merchan, A. F.; Maravelias, C. T. Reformulations of Mixed-Integer Programming ContinuousTime Models for Chemical Production Scheduling. Ind. Eng. Chem. Res. 2014, 53 (24), $10155-10165$.

8. Sundaramoorthy, A.; Maravelias, C. T. Computational Study of Network-Based MixedInteger Programming Approaches for Chemical Production Scheduling. Ind. Eng. Chem. Res. 2011, 50 (9), 5023-5040.

9. Carlier, J.; Pinson, E. An Algorithm for Solving the Job-Shop Problem. Manage Sci 1989, 35 (2), 164-176.

10. Adams, J.; Balas, E.; Zawack, D. The Shifting Bottleneck Procedure for Job Shop Scheduling. Manage Sci 1988, 34 (3), 391-401.

11. Baptiste, P.; Le Pape, C.; Nuijten, W. Constraint-based scheduling : applying constraint programming to scheduling problems. Kluwer Academic: Boston, 2001; p xii, 198 p.

12. Hooker, J. N. Logic, optimization, and constraint programming. INFORMS Journal on Computing 2002, 14 (4), 295-321.

13. Panek, S.; Engell, S.; Subbiah, S.; Stursberg, O. Scheduling of multi-product batch plants based upon timed automata models. Comput Chem Eng 2008, 32 (1-2), 275-291.

14. Subbiah, S.; Schoppmeyer, C.; Engell, S. An Intuitive and Efficient Approach to Process Scheduling with Sequence-Dependent Changeovers Using Timed Automata Models. Ind. Eng. Chem. Res. 50 (9), 5131-5152.

15. Mendez, C. A.; Cerda, J.; Grossmann, I. E.; Harjunkoski, I.; Fahl, M. State-of-the-art review of optimization methods for short-term scheduling of batch processes. Comput Chem Eng 2006, 30 (6-7), 913-946.

16. Maravelias, C. T. General framework and modeling approach classification for chemical production scheduling. AlChE J. 2012, 58 (6), 1812-1828.

17. Hooker, J. Logic-based methods for optimization : combining optimization and constraint satisfaction. John Wiley \& Sons: New York, 2000; p xvi, 495 p.

18. Jain, V.; Grossmann, I. E. Algorithms for hybrid MILP/CP models for a class of optimization problems. INFORMS Journal on Computing 2001, 13 (4), 258-276.

19. Harjunkoski, I.; Maravelias, C. T.; Bongers, P.; Castro, P. M.; Engell, S.; Grossmann, I. E.; Hooker, J.; Méndez, C.; Sand, G.; Wassick, J. Scope for industrial applications of production scheduling models and solution methods. Comput Chem Eng 2014, 62 (0), 161-193.

20. Ku, H. M.; Karimi, I. A. Scheduling in Serial Multiproduct Batch Processes with Finite Interstage Storage - A Mixed Integer Linear Program Formulation. Ind. Eng. Chem. Res. 1988, 27 (10), 1840-1848.

21. Pinto, J. M.; Grossmann, I. E. A Continuous-Time Mixed-Integer Linear-Programming Model for Short-Term Scheduling of Multistage Batch Plants. Ind. Eng. Chem. Res. 1995, 34 (9), 3037-3051. 
22. Cerda, J.; Henning, G. P.; Grossmann, I. E. A mixed-integer linear programming model for short-term scheduling of single-stage multiproduct batch plants with parallel lines. Ind. Eng. Chem. Res. 1997, 36 (5), 1695-1707.

23. Castro, P. M.; Grossmann, I. E. New continuous-time MILP model for the short-term scheduling of multistage batch plants. Ind. Eng. Chem. Res. 2005, 44 (24), 9175-9190.

24. Prasad, P.; Maravelias, C. T. Batch selection, assignment and sequencing in multi-stage multi-product processes. Comput Chem Eng 2008, 32 (6), 1106-1119.

25. Sundaramoorthy, A.; Maravelias, C. T. Modeling of storage in batching and scheduling of multistage processes. Ind. Eng. Chem. Res. 2008, 47 (17), 6648-6660.

26. Kondili, E.; Pantelides, C. C.; Sargent, R. W. H. A General Algorithm for Short-Term Scheduling of Batch-Operations .1. Milp Formulation. Comput Chem Eng 1993, 17 (2), 211227.

27. Pantelides, C. C. In Unified Frameworks for Optimal Process Planning and Scheduling, 2nd Conference on Foundations of Computer Aided Process Operations, Snowmass, CO, 1994; CACHE Publications: Snowmass, CO, 1994; pp 253-274.

28. Sundaramoorthy, A.; Maravelias, C. T. A General Framework for Process Scheduling. AlChE J. 2011, 57 (3), 695-710.

29. Velez, S.; Maravelias, C. T. Mixed-Integer Programming Model and Tightening Methods for Scheduling in General Chemical Production Environments. Ind. Eng. Chem. Res. 2013, 52 (9), 3407-3423.

30. Kelly, J. D.; Zyngier, D., Multi-product inventory logistics modeling in the process industries In Optimization and logistics challenges in the enterprise Chaovalitwongse, W.; Furman, K. C.; Pardalos, P. M., Eds. Springer: New York, 2009; pp 61-95.

31. Castro, P. M.; Harjunkoski, I.; Grossmann, I. E. Optimal scheduling of continuous plants with energy constraints. Comput Chem Eng 2011, 35 (2), 372-387.

32. Gimenez, D. M.; Henning, G. P.; Maravelias, C. T. A novel network-based continuous-time representation for process scheduling: Part I. Main concepts and mathematical formulation. Comput Chem Eng 2009, 33 (9), 1511-1528.

33. Gimenez, D. M.; Henning, G. P.; Maravelias, C. T. A novel network-based continuous-time representation for process scheduling: Part II. General framework. Comput Chem Eng 2009, 33 (10), 1644-1660.

34. Shah, N.; Pantelides, C. C.; Sargent, R. W. H. A General Algorithm for Short-Term Scheduling of Batch-Operations .2. Computational Issues. Comput Chem Eng 1993, 17 (2), 229-244.

35. Zhang, X.; Sargent, R. W. H. The optimal operation of mixed production facilities - A general formulation and some approaches for the solution. Comput Chem Eng 1996, 20 (6-7), 897904.

36. Schilling, G.; Pantelides, C. C. A simple continuous-time process scheduling formulation and a novel solution algorithm. Comput Chem Eng 1996, 20, S1221-S1226.

37. Mockus, L.; Reklaitis, G. V. Continuous time representation approach to batch and continuous process scheduling. 1. MINLP formulation. Ind. Eng. Chem. Res. 1999, 38 (1), 197-203.

38. Castro, P.; Barbosa-Povoa, A. P. F. D.; Matos, H. An improved RTN continuous-time formulation for the short-term scheduling of multipurpose batch plants. Ind. Eng. Chem. Res. 2001, 40 (9), 2059-2068.

39. Maravelias, C. T.; Grossmann, I. E. New general continuous-time state-task network formulation for short-term scheduling of multipurpose batch plants. Ind. Eng. Chem. Res. 2003, 42 (13), 3056-3074.

40. Sundaramoorthy, A.; Karimi, I. A. A simpler better slot-based continuous-time formulation for short-term scheduling in multipurpose batch plants. Chem Eng Sci 2005, 60 (10), 26792702.

41. Ierapetritou, M. G.; Floudas, C. A. Effective continuous-time formulation for short-term scheduling. 1. Multipurpose batch processes. Ind. Eng. Chem. Res. 1998, 37 (11), 4341-4359. 
42. Janak, S. L.; Lin, X. X.; Floudas, C. A. Enhanced continuous-time unit-specific event-based formulation for short-term scheduling of multipurpose batch processes: Resource constraints and mixed storage policies. Ind. Eng. Chem. Res. 2004, 43 (10), 2516-2533.

43. Susarla, N.; Li, J.; Karimi, I. A. A novel approach to scheduling multipurpose batch plants using unit-slots. AlChE J. 2010, 56 (7), 1859-1879.

44. Blomer, F.; Gunther, H. O. LP-based heuristics for scheduling chemical batch processes. International Journal of Production Research 2000, 38 (5), 1029-1051.

45. Sundaramoorthy, A.; Maravelias, C. T. Simultaneous batching and scheduling in multistage multiproduct processes. Ind. Eng. Chem. Res. 2008, 47 (5), 1546-1555.

46. Burkard, R. E.; Hatzl, J. Review, extensions and computational comparison of MILP formulations for scheduling of batch processes. Comput Chem Eng 2005, 29 (8), 1752-1769.

47. Janak, S. L.; Floudas, C. A. Improving unit-specific event based continuous-time approaches for batch processes: Integrality gap and task splitting. Comput Chem Eng 2008, 32 (4-5), 913-955.

48. Sahinidis, N. V.; Grossmann, I. E. Reformulation of multiperiod MILP models for planning and scheduling of chemical processes. Comput Chem Eng 1991, 15 (4), 255-272.

49. Yee, K. L.; Shah, N. Improving the efficiency of discrete time scheduling formulation. Comput Chem Eng 1998, 22, Supplement 1 (0), S403-S410.

50. Papageorgiou, L. G.; Pantelides, C. C. Optimal campaign planning scheduling of multipurpose batch semicontinuous plants .2. A mathematical decomposition approach. Ind. Eng. Chem. Res. 1996, 35 (2), 510-529.

51. Bassett, M. H.; Pekny, J. F.; Reklaitis, G. V. Decomposition techniques for the solution of large-scale scheduling problems. AlChE J. 1996, 42 (12), 3373-3387.

52. Kelly, J. D.; Zyngier, D. Hierarchical decomposition heuristic for scheduling: Coordinated reasoning for decentralized and distributed decision-making problems. Comput Chem Eng 2008, 32 (11), 2684-2705.

53. Maravelias, C. T.; Grossmann, I. E. Minimization of the makespan with a discrete-time statetask network formulation. Ind. Eng. Chem. Res. 2003, 42 (24), 6252-6257.

54. Wu, D.; Ierapetritou, M. G. Decomposition approaches for the efficient solution of short-term scheduling problems. Comput Chem Eng 2003, 27 (8-9), 1261-1276.

55. Calfa, B. A.; Agarwal, A.; Grossmann, I. E.; Wassick, J. M. Hybrid Bilevel-Lagrangean Decomposition Scheme for the Integration of Planning and Scheduling of a Network of Batch Plants. Ind. Eng. Chem. Res. 2013, 52 (5), 2152-2167.

56. Dimitriadis, A. D.; Shah, N.; Pantelides, C. C. RTN-based rolling horizon algorithms for medium term scheduling of multipurpose plants. Comput Chem Eng 1997, 21, S1061-S1066.

57. Lin, X. X.; Floudas, C. A.; Modi, S.; Juhasz, N. M. Continuous-time optimization approach for medium-range production scheduling of a multiproduct batch plant. Ind. Eng. Chem. Res. 2002, 41 (16), 3884-3906.

58. Mendez, C. A.; Cerda, J. Dynamic scheduling in multiproduct batch plants. Comput Chem Eng 2003, 27 (8-9), 1247-1259.

59. Roslof, J.; Harjunkoski, I.; Bjorkqvist, J.; Karlsson, S.; Westerlund, T. An MILP-based reordering algorithm for complex industrial scheduling and rescheduling. Comput Chem Eng 2001, 25 (4-6), 821-828.

60. Kopanos, G. M.; Mendez, C. A.; Puigjaner, L. MIP-based decomposition strategies for largescale scheduling problems in multiproduct multistage batch plants: A benchmark scheduling problem of the pharmaceutical industry. Eur J Oper Res 2010, 207 (2), 644-655.

61. Harjunkoski, I.; Grossmann, I. E. Decomposition techniques for multistage scheduling problems using mixed-integer and constraint programming methods. Comput Chem Eng 2002, 26 (11), 1533-1552.

62. Maravelias, C. T. A decomposition framework for the scheduling of single- and multi-stage processes. Comput Chem Eng 2006, 30 (3), 407-420.

63. Maravelias, C. T.; Grossmann, I. E. A hybrid MILP/CP decomposition approach for the continuous time scheduling of multipurpose batch plants. Comput Chem Eng 2004, 28 (10), 1921-1949. 
64. Roe, B.; Papageorgiou, L. G.; Shah, N. A hybrid MILP/CLP algorithm for multipurpose batch process scheduling. Comput Chem Eng 2005, 29 (6), 1277-1291.

65. Subrahmanyam, S.; Kudva, G. K.; Bassett, H. H.; Pekny, J. F. Application of distributed computing to batch plant design and scheduling. AlChE J. 1996, 42 (6), 1648-1661.

66. Ferris, M. C.; Maravelias, C. T.; Sundaramoorthy, A. Simultaneous Batching and Scheduling Using Dynamic Decomposition on a Grid. INFORMS Journal on Computing 2009, 21 (3), 398410.

67. Velez, S.; Maravelias, C. T. A branch-and-bound algorithm for the solution of chemical production scheduling MIP models using parallel computing. Comput Chem Eng 2013, 55 (0), 28-39.

68. Velez, S.; Maravelias, C. T. Advances in mixed-integer programming methods for chemical production scheduling. Annu Rev Chem Biomol Eng 2014, 5 (1), 97-121.

69. Velez, S.; Maravelias, C. T. Theoretical framework for formulating MIP scheduling models with multiple and non-uniform discrete-time grids. Comput Chem Eng 2015, 72 (0), 233254.

70. Barber, C. B.; Dobkin, D. P.; Huhdanpaa, H. The Quickhull algorithm for convex hulls. Acm T Math Software 1996, 22 (4), 469-483.

71. Papageorgiou, L. G.; Pantelides, C. C. Optimal campaign planning scheduling of multipurpose batch semicontinuous plants .1. Mathematical formulation. Ind. Eng. Chem. Res. 1996, 35 (2), 488-509.

72. Kallrath, J. Planning and scheduling in the process industry. Or Spectrum 2002, 24 (3), 219 250.

73. Wang, S.; Guignard, M. Hybridizing discrete- and continuous-time models for batch sizing and scheduling problems. Computers \& Operations Research 2006, 33 (4), 971-993.

74. Vooradi, R.; Shaik, M. A.; Gupta, N. M. Three-index Model for Westenberger-Kallrath Benchmark Scheduling Problem. Aip Conf Proc 2010, 1298, 380-385.

75. Vooradi, R.; Shaik, M. A. Improved three-index unit-specific event-based model for shortterm scheduling of batch plants. Comput Chem Eng 2012, 43, 148-172.

76. Nie, Y.; Biegler, L. T.; Wassick, J. M.; Villa, C. M. Extended Discrete-Time Resource Task Network Formulation for the Reactive Scheduling of a Mixed Batch/Continuous Process. Ind. Eng. Chem. Res. 2014. 